\title{
Transport of parallel momentum by collisionless drift wave turbulence
}

\author{
Diamond, P.H.; McDevitt, C.J.; Gürcan, O.D.; Hahm, T.S.; Naulin, Volker
}

Published in:

Physics of Plasmas

Link to article, DOI:

$10.1063 / 1.2826436$

Publication date:

2008

Document Version

Publisher's PDF, also known as Version of record

Link back to DTU Orbit

Citation (APA):

Diamond, P. H., McDevitt, C. J., Gürcan, O. D., Hahm, T. S., \& Naulin, V. (2008). Transport of parallel momentum by collisionless drift wave turbulence. Physics of Plasmas, 15(1), 012303.

https://doi.org/10.1063/1.2826436

\section{General rights}

Copyright and moral rights for the publications made accessible in the public portal are retained by the authors and/or other copyright owners and it is a condition of accessing publications that users recognise and abide by the legal requirements associated with these rights.

- Users may download and print one copy of any publication from the public portal for the purpose of private study or research.

- You may not further distribute the material or use it for any profit-making activity or commercial gain

- You may freely distribute the URL identifying the publication in the public portal

If you believe that this document breaches copyright please contact us providing details, and we will remove access to the work immediately and investigate your claim. 


\title{
Transport of parallel momentum by collisionless drift wave turbulence
}

\author{
P. H. Diamond, C. J. McDevitt, and Ö. D. Gürcan \\ Center for Astrophysics and Space Sciences, University of California, San Diego, \\ 9500 Gilman Drive, La Jolla, California 92093-0424, USA \\ T. S. Hahm \\ Princeton Plasma Physics Laboratory, Princeton University, Princeton, New Jersey 08543-0451, USA \\ V. Naulin \\ Riso National Laboratory, Danish Technical University, EURATOM Association, \\ DK-4000 Roskilde, Denmark
}

(Received 22 October 2007; accepted 28 November 2007; published online 8 January 2008)

\begin{abstract}
This paper presents a novel, unified approach to the theory of turbulent transport of parallel momentum by collisionless drift waves. The physics of resonant and nonresonant off-diagonal contributions to the momentum flux is emphasized, and collisionless momentum exchange between waves and particles is accounted for. Two related momentum conservation theorems are derived. These relate the resonant particle momentum flux, the wave momentum flux, and the refractive force. A perturbative calculation, in the spirit of Chapman-Enskog theory, is used to obtain the wave momentum flux, which contributes significantly to the residual stress. A general equation for mean $k_{\|}\left(\left\langle k_{\|}\right\rangle\right)$is derived and used to develop a generalized theory of symmetry breaking. The resonant particle momentum flux is calculated, and pinch and residual stress effects are identified. The implications of the theory for intrinsic rotation and momentum transport bifurcations are discussed. () 2008 American Institute of Physics. [DOI: 10.1063/1.2826436]
\end{abstract}

\section{INTRODUCTION}

Momentum transport, in particular, the turbulent transport of toroidal momentum has long been of interest to researchers in magnetic fusion and tokamak dynamics. The earliest investigations of toroidal momentum confinement were motivated by the desire to understand neutral beam injection performance and to probe the basic physics of confinement processes by comparing thermal and momentum diffusivities, $\chi_{i}, \chi_{\phi}$, etc. ${ }^{1}$ Subsequently, the dual realizations that electric field shear is a key control parameter for enhanced confinement (via turbulence suppression ${ }^{2}$ ) and that toroidal rotation "fed back" on electric field shear via radial force balance sparked ${ }^{3}$ further interest in toroidal momentum transport and its interplay with confinement. ${ }^{4}$ This growth in interest was accompanied by the developing realization that both self-generated (via Reynolds stress ${ }^{5}$ ) and externally driven flows contributed to electric field shear $v_{E}^{\prime}$. Key experiments included the discovery of the VH-mode, ${ }^{6}$ in which toroidal velocity shear regulated itself and other confinement channels via $v_{E}^{\prime}$, the discovery that a "pinch" or inward convection in the flux of toroidal momentum was required to model momentum profile structure ${ }^{7}$ (much as a particle pinch is required to explain density profile structure), and studies of the fluctuation-driven parallel Reynolds stress $\left\langle\widetilde{v}_{r} \widetilde{v}_{\|}\right\rangle$and its relation to local flow profile evolution. ${ }^{8}$

Interest in toroidal momentum transport was further stimulated by the discovery of "spontaneous" or "intrinsic" rotation ${ }^{9}$ and the realization that such intrinsic rotation may be required to suppress resistive wall modes in ITER, ${ }^{10}$ where neutral beam injection (NBI) is of limited utility and high cost. Spontaneous rotation refers to the observation that tokamak plasmas appear to rotate toroidally, at quite healthy velocities, in the absence of any apparent toroidal momentum input. The effort to understand spontaneous rotation has two thrusts, namely:

(i) An effort to understand nondiffusive, off-diagonal contributions to the momentum flux which may account for radially inward transport of momentum and resulting rotation profile peaking on axis.

(ii) An effort to understand the role of edge plasma dynamics in the onset of spontaneous rotation. In particular, several observations of the rotation evolution suggest that the profile of spontaneous rotation evolves inward, from the separatrix, so the edge may constitute a "dynamic" boundary condition or (possibly) a source.

As part of (ii), there has been intense interest in uncovering a possible fluctuation-driven momentum pinch or inward stress and relating it to the overall profile self-organization structure and energetics of the plasma. ${ }^{11}$ Efforts in this vein have discovered an inward convective pinch by perturbation experiments ${ }^{12}$ utilizing ripple loss with pulsed NBI, and have linked spontaneous rotation to the offset rotation values deduced from scans of rotation versus torque. ${ }^{13}$ As part of (ii), one striking observation which has emerged is that the Rice/ ITPA database scaling $\Delta v_{\phi}(0) \sim \Delta w_{p} / I_{p}$ is most clearly manifested in $\mathrm{H}$-mode plasmas, ${ }^{14}$ suggesting that an edge effect may be fundamental to its origin. Other edge plasma studies suggest a link of spontaneous rotation in the L-mode to scrape-off-layer flows (SOL), which can be manipulated by moving the divertor magnetic null-point location. ${ }^{15}$ This has lead to the speculation that the SOL flows may "spin-up" 
the core, but care must be taken with this interpretation of the data. Another striking feature of these experiments is the apparent disconnect between L-mode and H-mode spontaneous rotation phenomenology, suggesting that there may be an intimate link between transition dynamics (involving $v_{E}^{\prime}$, for example) and spontaneous rotation. Studies of the relation between edge pedestal structure and intrinsic rotation velocity scaling could be relevant here, but are conspicuous by their absence. Overall, a picture is emerging which suggests that in H-mode plasmas, intrinsic rotation builds up near the edge and is then redistributed inward, thus producing a peaked rotation profile. In addition, interesting studies on the TCV tokamak have also uncovered a new class of momentum transport bifurcations which occur in the core plasma. ${ }^{16}$ In a somewhat related vein, recent studies on the JT60-U tokamak also suggest that there is a departure from a simple diffusion-convection model of momentum transport in regions of steep $\nabla P$ located in the discharge core ${ }^{17}$ Generally, however, rather little is known about the interesting possibility of intrinsic rotation in torque-free, internal transport barrier (ITB) plasmas. Finally, virtually none of these studies take any serious account of the possibility of anomalous transport of poloidal momentum, as observed in JET. ${ }^{18}$ Poloidal rotation may be an especially important player at the edge or during an $\mathrm{L} \rightarrow \mathrm{H}$ transition.

Theoretical approaches to the problem of turbulent transport of toroidal momentum and intrinsic rotation have focused on attempts to calculate the various elements of the momentum flux. ${ }^{19}$ Various works include calculations of the momentum diffusivity $\chi_{\phi},{ }^{20}$ calculations of the momentum convection velocity $V$ in various models, ${ }^{21-24}$ and calculations of the residual stress driven by fluctuations and $\nabla P$, which can act as a local anomalous momentum source. ${ }^{25}$ Most of the calculations of off-diagonal flux elements involve some assumption of a mechanism for broken $k_{\|}$spectral symmetry, since $\left\langle\widetilde{v}_{r} \widetilde{v}_{\|}\right\rangle \sim\left\langle\nabla_{y} \widetilde{\phi} \nabla_{\|} \widetilde{\phi}\right\rangle$ requires $\left\langle k_{\|}\right\rangle_{\text {eff }} \neq 0$ for a significant nondiffusive component. In one case, such symmetry breaking occurred via an interesting interplay of curvature coupling and ballooning structure. ${ }^{23}$ In scenarios involving the electric field shear, progress was facilitated by drawing upon previous results for the effect of shear on turbulence and transport. ${ }^{26-29}$ Other approaches have invoked the effects of blobs or other coherent structures. ${ }^{30}$ Most of the calculations implemented so far have been extremely simple and based on fluid models. Even the few kinetic calculations have not treated the response of both resonant and nonresonant particles and have not addressed parallel acceleration effects. However, the general structure of a kinetic model has been discussed, to some extent, in Ref. 31 .

This paper presents a novel unified approach to the theory of turbulent transport of parallel (i.e., $\sim$ toroidal) momentum by collisionless drift waves. There are two principal motivations for yet another new look at this very old problem. These are:

(i) The need to identify and understand off-diagonal contributions to the turbulent momentum flux in collisionless drift wave turbulence. Such off-diagonal terms are not limited to convection (i.e., a "pinch"), but may also involve a residual stress, i.e., an element of the momentum flux not proportional to $\left\langle v_{\phi}\right\rangle$ or $\partial\left\langle v_{\phi}\right\rangle / \partial r$. Also in a collisionless plasma, off-diagonal terms also can conflate momentum transport with momentum transfer between resonant particles and waves.

(ii) The need to address (i) within a theoretical framework which accounts for wave-particle momentum exchange and the somewhat related problem of distinguishing between resonant and nonresonant transport. ${ }^{32}$ Confronting the latter is crucial for understanding momentum transport in a stationary plasma, where the quasilinear theory of nonresonant transport is no longer meaningful, but where significant momentum transport may occur via waves.

The phenomenology which drives the interest in off-diagonal contributions to the momentum flux, such as a possible inward convection (i.e., momentum "pinch") and a possible $\nabla P$-driven residual stress, has been discussed above. The considerations which drive motivation (ii) become apparent upon comparison of the simple physics of turbulent momentum transport to the more familiar phenomena of turbulent transport of particles and energy. Unlike density, momentum can be exchanged between waves and particles via resonant interaction with the underlying turbulence. Unlike the thermal energy density, which always exceeds the wave energy density (i.e., $\left.\varepsilon_{\omega} \ll n T\right)$ since $\{\tilde{n} / n, e \widetilde{\Phi} / T\} \ll 1$, the nonresonant particle momentum density of the fluctuations is in fact equal to the wave momentum density for electrostatic drift wave turbulence, since the field momentum density of the latter is negligible. This momentum equality has obvious implications for the onset of intrinsic rotation. Hence while transport theories may often neglect wave-particle energy exchange (though electron-ion energy coupling via fluctuations has been studied), and wave energy transport, they cannot ignore wave-particle momentum exchange or transport of wave momentum. Thus,

(i) Wave-particle momentum transfer is necessarily a key element of the momentum transport process, since "wave momentum" is equal to nonresonant particle momentum and can be exchanged with resonant particles.

(ii) The distinction between resonant and nonresonant transport processes is critical, since the former is mediated primarily by particles and likely is dominant near marginality in stiff profile regimes, while the latter is mediated by waves and likely is dominant in regimes of strong turbulence. A complete theory must address both processes.

Since nonresonant transport is mediated primarily by waves, it seems appropriate to describe it in terms of wave dynamics, quantities, populations, etc. The strong coupling between momentum transport and momentum transfer is readily appreciated upon a moments consideration of the mean field (i.e., quasilinear) evolution equation for the mean distribution function $\langle f\rangle$. This may easily be derived by averaging the drift/gyrokinetic equation 


$$
\frac{\partial f}{\partial t}+v_{\|} \nabla_{\|} f-\frac{c}{B} \nabla \Phi \times \hat{\mathbf{b}} \cdot \nabla f+\frac{e}{m} E_{\|} \frac{\partial f}{\partial v_{\|}}=0
$$

to obtain

$$
\begin{aligned}
\frac{\partial\langle f\rangle}{\partial t}= & \frac{1}{r} \frac{\partial}{\partial r}\left[r\left\{D_{r, r} \frac{\partial}{\partial r}\langle f\rangle+D_{r, v_{\|}} \frac{\partial\langle f\rangle}{\partial v_{\|}}\right\}\right] \\
& +\frac{\partial}{\partial v_{\|}}\left\{D_{v_{\|}, r} \frac{\partial\langle f\rangle}{\partial r}+D_{v_{\|}, v_{\|}} \frac{\partial\langle f\rangle}{\partial v_{\|}}\right\} .
\end{aligned}
$$

Here $D_{r, r}$ accounts for the familiar cross-field, scattering, and transport process, and $D_{v_{\|}, v_{\|}}$is simply the stochastic acceleration along the mean magnetic field. More interesting indeed, however, are the cross-terms $D_{r, v_{\|}}$and $D_{v_{\|}, r}$ which represent correlated radial scattering and parallel acceleration. Within the quasilinear approximation, these are

$$
D_{r, v_{\|} \approx} \sum_{\mathbf{k}} c_{s}^{2} \rho_{s}^{2} k_{\theta} k_{\|}\left|\frac{e \tilde{\Phi}}{T_{e}}\right|^{2} \Omega_{i} R\left(\omega-k_{\|} v_{\|}\right),
$$

where $R\left(\omega-k_{\|} v_{\|}\right)$is the response function. $D_{r, v_{\|}}$is striking in that

(i) It describes, within kinetic theory, precisely the process of correlated radial scattering and parallel acceleration which is required to generate a sheared parallel flow, as suggested by the phenomenon of intrinsic rotation.

(ii) It is explicitly proportional to the correlation $\left\langle\nabla_{\theta} \Phi \nabla_{\|} \Phi\right\rangle$, as is the Reynolds stress $\left\langle\widetilde{v}_{r_{E \times B}} \widetilde{v}_{\|}\right\rangle$, which is responsible for regulating radial transport of parallel momentum. Note that the sign of this correlator, and of $D_{r, v_{\|}}$can be either positive or negative, and so can produce a net inward or outward momentum flux.

(iii) Wave-particle resonance provide a direct way for obtaining for $D_{r, v_{\|}} \neq 0$. In particular, in contrast to speculations advanced in the course of previous studies, neither electric field shear nor toroidicity are necessarily required for off-diagonal momentum flux contributions.

A second motivation for this work is the long standing question of how to reconcile nonresonant transport with stationary turbulence. This question is often overlooked by transport models. Many important drift instabilities, such as the ion temperature gradient driven (ITG) mode, can have a strongly nonresonant, fluid-like character, particularly in regimes far from marginal stability. This observation naturally motivates the question of how to calculate the stationary nonlinear counterpart to this intrinsically nonstationary process. The traditional procedure of replacing the factor of $\left|\gamma_{k}\right|$ (or, incorrectly, $\gamma_{k}$ ) in the quasilinear theory with a decorrelation rate ignores the fact that for electrostatic turbulence, nonresonant particle dynamics are closely linked to wave dynamics, and so should be described in terms of the wave population, and other quantities characteristic of wave dynamics. In particular, consideration of momentum transport leads us to realize that since nonresonant particle momentum is the same as the wave momentum, then transport of nonresonant particle momentum is most naturally described as a process of transport of wave momentum. Another key issue here is the physics of the irreversibility which underlies the wave momentum transport. Thus, a major focus of this paper is the formulation of the calculation of the stationary state counterpart of the nonresonant particle momentum flux in terms of the properties and dynamics of the wave population density. As we will see, this formulation bares certain similarities to the structure of the theory of radiation hydrodynamics in the limit of large optical depth. ${ }^{33}$ The approach and results may also be of interest in the context of other problems involving mesoscale dynamics, most notably that of turbulence spreading. ${ }^{34-37}$

In this paper, then, we present a general theory of toroidal momentum transport by collisionless drift wave turbulence, with special emphasis on the physics of both nonresonant and resonant off-diagonal contributions to the momentum flux. Starting from the fundamental gyrokinetic equation, we derive a momentum theorem (MT-I) relating momentum evolution to the resonant particle momentum flux and the wave momentum flux. We extend this theorem, derived using a wave-energy balance relation (i.e., generalized Poynting theorem), to one which includes the effect of forces arising from the recoil on the plasma dielectric medium due to wave refraction (i.e., bending of wave rays must exert a force on the plasma!). This more general momentum theorem (MT-II) is derived using the full wave-kinetic equation. We then discuss the varieties of stationary momentum balance, and the possible interplay between the resonant particle momentum flux, the wave momentum flux and the refractive force, along with the significance of these for intrinsic rotation. After this, we undertake a perturbative calculation, in the spirit of Chapman-Enskog theory, to relate the wave momentum flux to the structure of the mean macroscopic wave quanta density distribution in both $\mathbf{x}$ and $\mathbf{k}$. The spatial and wave vector gradient dependence of this flux arise from spatial propagation and shear flow-induced wave wind-up. A generalized equation for the evolution of the mean parallel wave vector $\left\langle k_{\|}\right\rangle$is derived from the wave kinetic equation, and used to quantify the possible scenarios for competition between a number of possible and relevant symmetry breaking mechanisms. Of course, the resonant particle momentum transport is calculated as well, and shown to consist of the sum of a diffusive flux, a convective flux (a pinch, i.e., inward convection, in certain cases), and a residual stress contribution. The interplay among these is strongly sensitive to the mechanism of symmetry breaking. Finally, we consider the "bottom line" implications of the theory for intrinsic rotation and momentum transport bifurcations in tokamaks. In particular, we propose a novel class of momentum transport bifurcations and discuss the relation of these to the more general phenomenon of intrinsic rotation in $\mathrm{H}$-mode plasmas.

The remainder of this paper is organized as follows: In Sec. II, we prove two momentum conservation theorems at the level of mean field theory. The first theorem is a special, albeit simpler and more accessible case of the second. Section III presents a calculation of the radial flux of parallel wave momentum. A related approach allows the derivation of a general evolution equation for the net parallel quasipar- 
ticle momentum $\left(\int d \mathbf{k} k_{\|} N\right)$, which enables the quantitative comparison of different, competing symmetry breaking mechanisms. Section IV presents the calculation of the resonant particle momentum flux. In Sec. V, we discuss the theoretical results in the light of known aspects of momentum transport phenomenology. Special attention is devoted to theoretical predictions of various types of momentum transport bifurcations. This section is useful for those who are mainly interested in applications rather than development of the theory. Section VI presents a discussion and conclusions.

\section{MEAN FIELD THEORY AND CONSERVATION OF MOMENTUM IN COLLISIONLESS DRIFT WAVE TURBULENCE}

In this section we discuss conservation of momentum and the structure of the momentum budget relation in the mean field theory of drift wave turbulence. We assume a simple geometry $r, \theta, \phi$ and leave extensions to the torus to a future paper. Two theorems of conservation of momentum (MT-I, MT-II) are proved. The latter (MT-II) constitutes a generalization of the former (MT-I). Special emphasis is placed on delineating the roles of resonant particles and waves in momentum balance and transport, as well as on the resolution of several questions related to how nonresonant diffusion effects are manifested in a stationary state.

A brute force approach to the problem of momentum balance and conservation might proceed as follows. Starting from the drift kinetic equation (i.e., for $k_{\perp} \rho_{i} \ll 1$ ) for ions in collisionless electrostatic turbulence,

$$
\frac{\partial f}{\partial t}+v_{\|} \nabla_{\|} f-\frac{c}{B} \nabla \Phi \times \hat{\mathbf{b}} \cdot \nabla f+\frac{e}{m} E_{\|} \frac{\partial f}{\partial v_{\|}}=0
$$

the mean field equation follows as

$$
\frac{\partial\langle f\rangle}{\partial t}+\frac{1}{r} \frac{\partial}{\partial r}\left[r\left\langle\widetilde{v}_{r} \tilde{f}\right\rangle\right]+\frac{\partial}{\partial v_{\|}}\left\langle\frac{e}{m} \tilde{E}_{\|} \tilde{f}\right\rangle=0
$$

so that the usual quasilinear calculation gives

$$
\begin{aligned}
\frac{\partial\langle f\rangle}{\partial t}= & \frac{1}{r} \frac{\partial}{\partial r}\left[r\left\{D_{r, r} \frac{\partial}{\partial r}\langle f\rangle+D_{r, v_{\|}} \frac{\partial\langle f\rangle}{\partial v_{\|}}\right\}\right] \\
& +\frac{\partial}{\partial v_{\|}}\left\{D_{v_{\|}, r} \frac{\partial\langle f\rangle}{\partial r}+D_{v_{\|}, v_{\|}} \frac{\partial\langle f\rangle}{\partial v_{\|}}\right\},
\end{aligned}
$$

where

$$
\begin{aligned}
& D_{r, r}=\rho_{s} c_{s} \sum_{\mathbf{k}} \rho_{s}^{2} k_{\theta}^{2}\left|\frac{e \widetilde{\Phi}}{T_{e}}\right|^{2} \Omega_{i} R\left(\omega-k_{\|} v_{\|}\right), \\
& D_{r, v_{\|}}=D_{v_{\|}, r}=c_{s}^{2} \sum_{\mathbf{k}} \rho_{s}^{2} k_{\theta} k_{\|}\left|\frac{e \widetilde{\Phi}}{T_{e}}\right|^{2} \Omega_{i} R\left(\omega-k_{\|} v_{\|}\right), \\
& D_{v_{\|}, v_{\|}}=c_{s}^{2} \Omega_{i} \sum_{\mathbf{k}} \rho_{s}^{2} k_{\|}^{2}\left|\frac{e \widetilde{\Phi}}{T_{e}}\right|^{2} \Omega_{i} R\left(\omega-k_{\|} v_{\|}\right)
\end{aligned}
$$

are the coefficients corresponding to radial spatial scattering by stochastic $E \times B$ drifts $\left(D_{r, r}\right)$, parallel stochastic acceleration as in the 1D Vlasov plasma $\left(D_{v_{\|,},}\right)$, and correlated par- allel acceleration and radial scattering $\left(D_{r, v}\right)$, which is related to the generation of a radially sheared parallel flow. Since plasma momentum $\left\langle P_{\|}\right\rangle=m_{i} \int d v_{\|} v_{\|}\langle f\rangle$ is carried by ions, taking the $v_{\|}$moment of Eq. (2a) says that parallel momentum evolves according to

$$
\begin{aligned}
\frac{\partial}{\partial t}\left\langle P_{\|}\right\rangle= & \frac{m_{i}}{r} \frac{\partial}{\partial r}\left[r \int d v_{\| i} v_{\| i}\left(D_{r, r} \frac{\partial\langle f\rangle_{i}}{\partial r}+D_{r, v_{\|}} \frac{\partial\langle f\rangle_{i}}{\partial v_{\|}}\right)\right] \\
& -m_{i} \int d v_{\| i}\left[D_{v_{\|}, r} \frac{\partial\langle f\rangle_{i}}{\partial r}+D_{v_{\|}, v_{\|}} \frac{\partial\langle f\rangle_{i}}{\partial v_{\|}}\right] .
\end{aligned}
$$

We see, then, that Eq. (3) has the form,

$$
\frac{\partial\left\langle P_{\|}\right\rangle}{\partial t}=-\frac{1}{r} \frac{\partial}{\partial r}\left[r \Pi_{r, \|}\right]+S_{\|}
$$

and states that momentum evolves via transport by turbulence induced stress $\left(\Pi_{r, \|}\right)$ and via a local "source or sink," due to wave-particle scattering (which we term momentum transfer). Note that the definitions of $\Pi_{r, \|}$ and $S_{\|}$in terms of fundamental microscopic quantities are clear from Eqs. (3) and (4).

The result of Eq. (4) is somewhat unsettling on account of the appearance of an explicit local fluctuation driven source $S_{\|}$for $\partial\langle f\rangle / \partial t$. Recall that the aim here is to determine the off-diagonal momentum flux (i.e., transport), in order to understand possible inward convection and stresses. The presence of a local, fluctuation induced source significantly complicates this task. One does not know how significant this effect is, though since $D_{r, v_{\|}} \sim D_{v_{\|}, r}, S_{\|}$is clearly not negligible, and neither does one know the fate of momentum coupled to/from the particles to the fluctuations. One thing that $i s$ clear, however, is that the mean field contributions to $\langle f\rangle$ evolution from parallel acceleration $\left(e / m\left\langle\tilde{E}_{\|} \partial \tilde{f} / \partial v_{\|}\right\rangle\right)$ should not be neglected a priori. Another is that a natural way to formulate the theory is in terms of resonant particles and waves, since these conserve momentum between them.

The physical content of $S_{\|}$in Eq. (4) is best resolved by reconsidering the basic energy and momentum conservation theorems for the quasilinear theory of the 1D Vlasov plasma. For that well-studied system, we know that energy balance may be stated in either of two equivalent ways: as conservation of the sum of resonant particle $\left(\varepsilon_{\mathrm{RP}}\right)$ and wave $\left(\varepsilon_{W}\right)$ energy densities, i.e.,

$$
\frac{d}{d t}\left(\varepsilon_{\mathrm{RP}}+\varepsilon_{W}\right)=0
$$

or as conservation of the sum of particle and electrostatic field energy density, i.e.,

$$
\frac{d}{d t}\left(\varepsilon_{P}+\varepsilon_{F}\right)=0 .
$$

The equivalence of these two results follows from the fact that $\varepsilon_{W}=\varepsilon_{F}+\varepsilon_{\mathrm{NRP}}$, i.e., waves (collective modes) are supported both by nonresonant particles and fields. We observe that Eq. (5a) can be alternatively recast in terms of the quasiparticle density $N$ (wave quanta density or usually action density) by noting that $\varepsilon_{W}=N(\mathbf{k}, \mathbf{x}, t) \omega_{\mathbf{k}}$, so that the sum of 
resonant particle energy and quasiparticle energy is conserved. $^{32}$

Similarly, for momentum, (in the absence of external sources) we can write

$$
\frac{d}{d t}\left(P_{\mathrm{RP}}+P_{W}\right)=0
$$

and

$$
\frac{d}{d t}\left(P_{P}+P_{F}\right)=0
$$

Here $P_{\mathrm{RP}}$ is the resonant particle momentum density, $P_{W}$ is the wave momentum density (sometimes referred to as the pseudomomentum density), and $P_{P}=P_{\mathrm{RP}}+P_{\mathrm{NRP}}$ is the total particle momentum density. Equation (6b) follows from Eq. (6a) since the field momentum $P_{F}$ for electrostatic turbulence is necessarily zero $\left(\widetilde{E} \times \widetilde{B} / 4 \pi c^{2}=0\right.$, since $\left.\widetilde{B}=0\right)$. Thus the increment of nonresonant particle momentum density is necessarily equal to the wave momentum density, i.e.,

$$
\delta P_{\mathrm{NRP}}=P_{W},
$$

where $\delta P_{\mathrm{NRP}}=P_{\mathrm{NRP}}-P_{\mathrm{NRP}}^{(0)}$ is the momentum density associated with the fluctuation driven nonresonant particle flow, and $P_{\mathrm{NRP}}^{(0)}$ is the zeroth order nonresonant particle momentum density, associated with the background (mean) flow. Equation (6c) is a simple, albeit important, identity which is applicable to any system of electrostatic turbulence, including electrostatic drift wave turbulence.

In this paper, we focus on electrostatic drift wave driven transport. Thus, turning to momentum conservation in 3D electrostatic drift wave turbulence, it seems incontrovertible that resonant particle momentum density will be conserved against wave momentum density. In contrast to the "textbook" 1D Vlasov problem, however, the radial fluxes of both quantities will enter, as well. Thus, we can expect a conservation relation of the form,

$$
\frac{\partial}{\partial t} t\left\langle P_{\|}\right\rangle_{\mathrm{RP}}+\frac{1}{r} \frac{\partial}{\partial r}\left[r\left\langle\widetilde{v}_{E} \widetilde{P}_{\|}\right\rangle_{\mathrm{RP}}\right]+\frac{\partial}{\partial t}\left\langle P_{\|}\right\rangle_{w}+\frac{1}{r} \frac{\partial}{\partial r}\left[r \Pi_{r, \|}^{w}\right]=0,
$$

where $\left\langle\widetilde{v}_{E} \widetilde{P}_{\|}\right\rangle$is the radial $E \times B$ flux of resonant particle parallel momentum and $\Pi_{r, \|}^{w}$ is the radial flux of parallel wave momentum. In a quasiparticle or eikonal formulation of wave dynamics, $\left\langle\Pi_{r, \|}^{w}\right\rangle=\int d k_{\|} v_{g r} k_{\|}\langle N\rangle$, where $v_{\text {gr }}$ is the radial group velocity of the waves. $^{38}$ Since, however, $P_{W}=\delta P_{\mathrm{NRP}}$, Eq. (7) immediately undergoes an important simplification to

$$
\frac{\partial}{\partial t}\left\langle\delta P_{\|}\right\rangle+\frac{1}{r} \frac{\partial}{\partial r}\left[r\left(\left\langle\widetilde{v}_{E r} \widetilde{P}_{\|}\right\rangle_{\mathrm{RP}}+\left\langle\Pi_{r, \|}^{w}\right\rangle\right)\right]=0 .
$$

At this point, a careful discussion of how $\left\langle\delta P_{\|}\right\rangle$(the mean momentum increment driven by the fluctuations) relates to the evolution of the background momentum density is necessary. The total momentum density $\left\langle P_{\|}\right\rangle$is defined as the sum of the momenta of the mean flow and that of the fluctuations. Thus, we have

$$
\left\langle P_{\|}\right\rangle=P_{\|, 0}+\left\langle\delta P_{\|}\right\rangle,
$$

where

$$
\frac{d\left\langle P_{\|}\right\rangle}{d t}=S
$$

i.e., we find it useful to introduce an external momentum density source $S(r, t)$ (i.e., such as NBI-driven momentum input). Thus,

$\frac{\partial}{\partial t}\left(P_{\|, 0}+\left\langle\delta P_{\|}\right\rangle\right)+\frac{1}{r} \frac{\partial}{\partial r}\left(r\left[\left\langle\Pi_{r, \|}^{\mathrm{NRP}}\right\rangle_{0}+\left\langle\widetilde{v}_{E r} \widetilde{P}_{\|}\right\rangle_{\mathrm{RP}}+\left\langle\Pi_{r, \|}^{w}\right\rangle\right]\right)=S$

follows as the dynamical evolution equation for total momentum. Here $\left\langle\Pi_{\mathrm{NR}}\right\rangle_{0}$ is the zeroth order (nonresonant) momentum flux which corresponds to the nonresonant quasilinear flux plus the neoclassical flux (i.e., the latter being the only surviving contributor to the flux in the absence of fluctuations). The quasilinear part is $O\left(\left|e \widetilde{\Phi} / T_{e}\right|^{2}\right)$ and is proportional to the magnitude of the linear growth rate, $\left|\gamma_{k}\right|$. This differs from the wave momentum flux in a subtle way. In this regard, and by way of contrast, note that $\left\langle\Pi_{r, \|}^{w}\right\rangle$ in contrast, need not be limited to a second order effect in fluctuation level, and can involve "spreading" of the momentum of fluctuations, and other nonlinear processes. Physically, $\left\langle\Pi_{r, \|}^{\mathrm{NRP}}\right\rangle_{0}$ is a necessary part of the description of the momentum density evolution in time, though it asymptotes to the (usually negligible) collisional level in the case of stationary turbulence.

Regarding temporal evolution, it is useful here to discuss the distinctions between "stationary mean momentum profile," "stationary turbulence" and "intrinsic rotation." Starting from Eq. (9c), stationarity of the mean momentum profile requires $\partial_{t}\left[P_{\|, 0}+\left\langle\delta P_{\|}\right\rangle\right]=0$, so the momentum is steady on transport time scales. This then gives the usual balance between momentum input and transport, i.e.,

$$
\frac{1}{r} \frac{\partial}{\partial r}\left(r\left[\left\langle\Pi_{r, \|}^{\mathrm{NRP}}\right\rangle_{0}+\left\langle\widetilde{v}_{E r} \widetilde{P}_{\|}\right\rangle_{\mathrm{RP}}+\left\langle\Pi_{r, \|}^{w}\right\rangle\right]\right)=S
$$

so the mean profile is determined by

$$
\left\langle\Pi_{r, \|}^{\mathrm{NRP}}\right\rangle_{0}+\left\langle\widetilde{v}_{E r} \widetilde{P}_{\|}\right\rangle_{\mathrm{RP}}+\left\langle\Pi_{r, \|}^{w}\right\rangle=\frac{1}{r} \int r^{\prime} d r^{\prime} S\left(r^{\prime}\right)
$$

and the profile boundary conditions. In addition, it is necessary to parametrize $\left\langle\Pi_{r, \|}^{w}\right\rangle$ in terms of plasma parameter profiles. Generally this need not be a purely local relation. Note that Eq. (10b) allows the possibility that the turbulence can be at least weakly nonstationary, though the profile is not globally evolving. For stationary turbulence, we have $\left\langle\Pi_{r, \|}^{\mathrm{NRP}}\right\rangle_{0} \rightarrow\left\langle\Pi_{r, \|}\right\rangle^{C}$, where $\left\langle\Pi_{r, \|}\right\rangle^{C}$ is the collisional momentum flux, so that Eq. (10b) becomes

$$
\left\langle\Pi_{r, \|}\right\rangle^{C}+\left\langle\widetilde{v}_{E r} \widetilde{P}_{\|}\right\rangle_{\mathrm{RP}}+\left\langle\Pi_{r, \|}^{w}\right\rangle=\frac{1}{r} \int r^{\prime} d r^{\prime} S\left(r^{\prime}\right)
$$

which is the usual stationary state (for both mean field and turbulence) criterion. Note that $\left\langle\Pi_{r, \|}^{w}\right\rangle$ accounts for the nonresonant transport in this stationary state, and that this trans- 
port is purely wave transport. Equation (10c) indicates that the most important results of this paper are the momentum theorems for the evolution of $\left\langle\delta P_{\|}\right\rangle$(i.e., MT-I, MT-II, proved in this section) and the actual calculations of $\left\langle\widetilde{v}_{E r} \widetilde{P}_{\|}\right\rangle_{\mathrm{RP}}$ and $\left\langle\Pi_{r, \|}^{w}\right\rangle$ (discussed in Secs. III and IV, respectively). Finally, the case of "intrinsic rotation" for stationary turbulence corresponds to one where $S \rightarrow 0$, i.e., where

$$
\left\langle\Pi_{r, \|}\right\rangle{ }^{C}+\left\langle\widetilde{v}_{E r} \widetilde{P}_{\|}\right\rangle_{\mathrm{RP}}+\left\langle\Pi_{r, \|}^{w}\right\rangle=0
$$

and which has nontrivial profile solutions in the absence of momentum input. Note that the boundary conditions for the solution of Eq. (10d) will have a significant impact on the answer to this question. The interesting possibility of $\langle S\rangle=0$ but $\tilde{S} \neq 0$, i.e., a momentum noise source due to avalanches, etc., ${ }^{39,40}$ will be discussed in a future paper. In particular (for $\langle\widetilde{S}\rangle=0$ ) it would be interesting to compare the intrinsic rotation profile for $\widetilde{S}=0$ with that calculated for small but finite $\tilde{S}$.

Equation (8) constitutes the first momentum conservation theorem (MT-I) to be presented in this study. Two salient features of Eq. (8) are

The somewhat ill-defined nonresonant particle momentum flux has been eliminated in favor of the wave-momentum flux $\left\langle\Pi_{r, \|}^{w}\right\rangle$, which is well defined for stationary turbulence. The irreversibility which underpins $\left\langle\Pi_{r, \|}^{w}\right\rangle$ in a stationary state must necessarily originate in nonlinear wave-wave and wave-particle interactions.

(ii) The ambiguous anomalous source $S_{\|}$which appears in Eq. (3) is replaced by $-\nabla \cdot\left\langle\hat{\mathbf{r}} \Pi_{r, \|}^{w}\right\rangle$, which suggests that the apparent "source or sink" due to wave-particle interaction must ultimately dispose of momentum by coupling it to radially propagating waves. This suggests that momentum transport is best described in terms of the combined flux of resonant particles and waves, since these two populations conserve momentum between them. This leads us naturally to think in terms of a picture of resonant particles and quasiparticles.

Points (i) and (ii) go a long way toward resolving the questions inherent in Eq. (3). The challenge, now, is to prove MT-I, as expressed by Eq. (8). We now turn to this task.

Momentum theorem I is most directly proved by relating evolution of net resonant particle momentum to the sum of the resonant particle momentum flux and the net force exerted on the plasma by the resonant particles. The latter can then be linked to wave-momentum, yielding a net momentum conservation theorem. The evolution equation for the total resonant particle momentum is derived by adding the parallel velocity moments of the individual resonant electron and resonant ion drift kinetic equations,

$$
\frac{\partial}{\partial t}\left\langle P_{\|}\right\rangle_{\mathrm{RP}}+\frac{1}{r} \frac{\partial}{\partial r}\left[r\left\langle\widetilde{v}_{E r} \widetilde{P}_{\|}\right\rangle_{\mathrm{RP}}\right]=e\left\langle\widetilde{E}_{\|}\left(\widetilde{n}_{i}-\widetilde{n}_{e}\right)\right\rangle_{\mathrm{RP}}
$$

The right-hand side of Eq. (11) does not vanish on account of quasineutrality, since $\widetilde{n}_{i}=\widetilde{n}_{e}$ is not equivalent to $\widetilde{n}_{i R}=\widetilde{n}_{e R}$, i.e., resonant ions need not be neutralized by resonant electrons (see Appendix A for the explicit evaluation of this term). It is convenient to express the density perturbations in terms of the susceptibilities $\chi_{i, e}\left(k, \omega_{k}\right)$ via

$$
\tilde{n}_{(i, e) \mathbf{k}, \omega_{\mathbf{k}}}=\frac{n_{0} e}{T_{e}} \widetilde{\Phi}_{\mathbf{k}, \omega_{\mathbf{k}}} \chi_{(i, e)}\left(\mathbf{k}, \omega_{\mathbf{k}}\right) .
$$

Note that $\chi_{(i, e)}$ refers to a response function, not a thermal diffusivity! Recalling that $\chi_{(i, e)}\left(\mathbf{k}, \omega_{\mathbf{k}}\right)$ for resonant particles is imaginary, we thus obtain

$$
\begin{aligned}
e\left\langle\widetilde{E}_{\|}\left(\widetilde{n}_{i}-\tilde{n}_{e}\right)\right\rangle= & -n_{0} T_{e} \sum_{\mathbf{k}} k_{\|}\left[\operatorname{Im} \chi_{i}\left(\mathbf{k}, \omega_{\mathbf{k}}\right)-\operatorname{Im} \chi_{e}\left(\mathbf{k}, \omega_{\mathbf{k}}\right)\right] \\
& \times\left|\frac{e \widetilde{\Phi}_{\mathbf{k}}}{T_{e}}\right|^{2} .
\end{aligned}
$$

This relates the net force density acting on resonant particles to their dielectric response functions. It is now convenient to recall the quasilinear wave-energy ("Poynting") theorem for drift waves,

$$
\frac{\partial}{\partial t} \varepsilon_{\omega_{\mathbf{k}}}+\nabla \cdot \mathbf{S}_{\mathbf{k}}+Q_{\mathbf{k}}=0
$$

i.e., that wave energy density $\varepsilon_{\omega_{\mathbf{k}}}$ evolves via divergence of the wave energy density flux $\mathbf{S}_{\mathbf{k}}$ and local dissipation $Q_{\mathbf{k}}$. Here, $\varepsilon_{\omega_{\mathbf{k}}}, \mathbf{S}_{\mathbf{k}}$, and $Q_{\mathbf{k}}$ are given, respectively, by

$$
\begin{aligned}
& \varepsilon_{\omega_{\mathbf{k}}}=n_{0} T_{e} \omega_{\mathbf{k}}\left(\frac{\partial \chi}{\partial \omega}\right)_{\omega_{\mathbf{k}}}\left|\frac{e \tilde{\Phi}}{T_{e}}\right|^{2}, \\
& \mathbf{S}_{\mathbf{k}}=-n_{0} T_{e} \omega_{\mathbf{k}}\left(\frac{\partial \chi}{\partial \mathbf{k}}\right)_{\omega_{\mathbf{k}}}\left|\frac{e \tilde{\Phi}}{T_{e}}\right|^{2}, \\
& Q_{\mathbf{k}}=-n_{0} T_{e} \omega_{\mathbf{k}}(\operatorname{Im}[\chi])_{\omega_{\mathbf{k}}}\left|\frac{e \tilde{\Phi}}{T_{e}}\right|^{2},
\end{aligned}
$$

where

$$
\chi(\mathbf{k}, \omega)=\chi_{i}(\mathbf{k}, \omega)-\chi_{e}(\mathbf{k}, \omega) .
$$

Equation (12) is a straightforward adaptation of a more general result for waves in a dielectric medium to the special case of drift waves, which we consider here. The result of Eqs. (12) and (13) is proved in Refs. 41 and 42. Using Eqs. (12) and (13), we immediately note that

$$
e\left\langle\widetilde{E}_{\|}\left(\widetilde{n}_{i}-\widetilde{n}_{e}\right)\right\rangle_{R}=\sum_{\mathbf{k}} \frac{k_{\|}}{\omega_{\mathbf{k}}} Q_{\mathbf{k}}=-\sum_{\mathbf{k}} \frac{k_{\|}}{\omega_{\mathbf{k}}}\left(\frac{\partial}{\partial t} \varepsilon_{\omega_{\mathbf{k}}}+\nabla \cdot \mathbf{S}_{\mathbf{k}}\right)
$$

thus directly relating the dielectric medium's force on resonant particles to the evolution of wave energy! It is no surprise to see the proportionality between resonant density imbalance $\left(\widetilde{n}_{i R}-\tilde{n}_{e R}\right)$ and $\nabla \cdot \mathbf{S}$ (the divergence of the wave energy density flux) emerge in this theory, since the latter includes radial wave propagation, which originates in the polarization charge contribution to quasineutrality, and which is required to neutralize $\widetilde{n}_{i R}-\widetilde{n}_{e R}$. Since the spatiotemporal evolutions of $\varepsilon_{\omega_{\mathbf{k}}}$ and $\mathbf{S}_{\mathbf{k}}$ are, by construction, 
"slow," i.e., corresponding to envelope scales, we have $\varepsilon_{\omega}^{-1} \partial \varepsilon_{\omega} / \partial t \ll \omega_{\mathbf{k}}$ and $|S|^{-1}\left|\nabla_{r} S\right|<|k|$, so

$$
e\left\langle\widetilde{E}_{\|}\left(\widetilde{n}_{i}-\widetilde{n}_{e}\right)\right\rangle_{R P}=-\sum_{\mathbf{k}}\left(\frac{\partial}{\partial t}\left\langle P_{\|}\right\rangle_{W}+\frac{1}{r} \frac{\partial}{\partial r}\left[r\left\langle\Pi_{r, \|}^{w}\right\rangle\right]\right) .
$$

Since $P_{\|}^{w}=\left(k_{\|} / \omega_{\mathbf{k}} h\right) \varepsilon_{\omega}$ and $\left(k_{\|} / \omega_{\mathbf{k}}\right) \mathbf{S}=\Pi^{w}$, consistent with the simple geometry assumed here, only radial variation of the envelope is considered. To complete the proof of (MT-I), we need to only combine Eq. (14) with Eq. (11), and note that $\left\langle P_{\|}\right\rangle_{\mathrm{RP}}+\left\langle P_{\|}\right\rangle_{W}=\left\langle\delta P_{\|}\right\rangle$, since wave momentum and nonresonant particle momentum are identical in the frame of the background plasma. These steps yield

$$
\frac{\partial\left\langle\delta P_{\|}\right\rangle}{\partial t}+\frac{1}{r} \frac{\partial}{\partial r}\left[r\left(\left\langle\widetilde{v}_{E r} \widetilde{P}_{\|}\right\rangle_{\mathrm{RP}}+\int d \mathbf{k} v_{g r} P_{\| \mathbf{k}}^{w}\right)\right]=0
$$

which proves the first momentum theorem. Here $P_{\| \mathbf{k}}^{w}$ is the parallel wave momentum density for the wave with the wave vector $\mathbf{k}$, and radial group velocity $v_{\mathrm{gr}}$. Note that Eq. (15) eliminates the local force in Eq. (11), in favor of the mean radial flux of parallel wave momentum $\left\langle\Pi_{r, \|}^{w}\right\rangle$. More generally, Eq. (15) relates the total, fluctuation-induced parallel force on the plasma to the sum of the fluxes of resonant particle and wave momentum. Of course, given the disparity between electron and ion inertia $\left(m_{e} \ll m_{i}\right),\left\langle P_{\|}\right\rangle$is carried primarily by ions. However we emphasize that no limiting assumptions concerning the electron response (i.e., "adiabatic" or " $\mathrm{i}-\delta$ ") were made in the course of this derivation.

The alert reader may notice that the result of Eq. (15) (i.e., MT-I) does not account for the force exerted on the plasma due to wave refraction. In particular, given sheared flows and other profile variations, drift wave trajectories will bend and refract, so the wave packet momentum will exhibit a concomitant change in direction. ${ }^{43,44}$ Of course, since the waves and dielectric media are (strongly) coupled, the process of wave refraction will induce a recoil force on the plasma. This refractive force will then necessarily change the plasma momentum, and thus should enter the momentum budget. ${ }^{45-47}$ A proper treatment of the refractive force requires an extension of MT-I. We note in passing that the force which drives the amplification of zonal flow shear is just the radial component of the refractive force, and in that case, the net power expended by the refractive force is precisely the rate of growth of zonal flow energy. The basic physics and energetics of the refractive force are discussed further in Appendix B. Here we focus on the derivation of a momentum conservation theorem which accounts for refraction effects (i.e., MT-II).

In order to derive MT-II, it is advantageous to start from the wave kinetic equation, which effectively constitutes a Boltzmann equation for the quasiparticle or wave quanta density $N(\mathbf{x}, \mathbf{k}, t)$, Here, in the context of simple drift wave models, such as extended Hasegawa-Mima models, the $(\mathbf{x}, \mathbf{k})$ phase space density which satisfies the wave kinetic equation is closely related to the potential enstrophy density $\Omega=\left(1+k_{\perp}^{2} \rho_{s}^{2}\right)^{2}\left|e \hat{\phi}_{\mathbf{k}} / T\right|^{2}, \quad$ since $\quad N(\mathbf{x}, \mathbf{k}, t)=\left(1+k_{\perp}^{2} \rho_{s}^{2}\right)^{2} I_{w}$, where the Wigner distribution function $I_{w}$ $=\int d \mathbf{q} e^{i \mathbf{q} \cdot \mathbf{x}}\left\langle\hat{\phi}_{\mathbf{k}+\mathbf{q}} \hat{\phi}_{-\mathbf{k}}\right\rangle$. The interpretation of $\Omega$ as a "quanta density" follows from the intuition that $\Omega$ may be viewed as a vortex or "roton" density. ${ }^{48-51}$ The wave kinetic equation is

$$
\frac{\partial N}{\partial t}+\nabla \cdot\left[\left(\mathbf{v}_{g}+\mathbf{V}\right) N\right]+\nabla_{\mathbf{k}} \cdot\left[-\frac{\partial}{\partial \mathbf{x}}(\omega+\mathbf{k} \cdot \mathbf{V}) N\right]=C(N),
$$

where the collision integral may be decomposed as

$$
C(N)=C_{w-w}(N)+C_{w-p}(N) .
$$

Here $C_{w-w}(N)$ corresponds to resonant nonlinear wave-wave interactions which do not conserve $N$, while $C_{w-p}(N)$ corresponds to resonant linear and nonlinear wave-particle interactions. Thus, for (generalized) three wave interaction processes arising from quadratic nonlinearity, we can expect $C_{w-w}(N)$ to have the form,

$$
\begin{aligned}
C_{w-w}(N)= & \sum_{\mathbf{k}^{\prime}}\left\{C_{1}\left(\mathbf{k}^{\prime}, \mathbf{k}-\mathbf{k}^{\prime}\right) N_{\mathbf{k}^{\prime}} N_{\mathbf{k}-\mathbf{k}^{\prime}}\right. \\
& \left.-C_{2}\left(\mathbf{k}, \mathbf{k}^{\prime}\right) N_{\mathbf{k}^{\prime}} N_{\mathbf{k}}\right\} \delta\left(\omega_{\mathbf{k}-\mathbf{k}^{\prime}}+\omega_{\mathbf{k}^{\prime}}-\omega_{\mathbf{k}}\right),
\end{aligned}
$$

where conservation of energy and momentum by the microscopic interaction processes require that

$$
\int d k\left(\begin{array}{c}
\mathbf{k} \\
\omega_{\mathbf{k}}
\end{array}\right) C_{w-w}(N)=0
$$

independent of the detailed structure of $C_{w-w}(N)$. Note that the Manley-Rowe relations ${ }^{52}$ state that while the microscopic interactions do conserve energy and momentum, they do not conserve quasiparticle number (apart from the important exception of induced diffusion $\left.{ }^{53}\right) C_{w-p}(N)$, which represents wave-particle interaction, is necessarily dissipative, since quasiparticles exchange energy and momentum with particles via linear and nonlinear resonant interaction. The lowest order contributions to the latter occurs, for example, via the familiar beat wave resonance associated with nonlinear Landau damping [i.e., when $\left.\omega+\omega^{\prime}=\left(k_{\|}+k_{\|}^{\prime}\right) v_{\|}\right]$.

In order to derive MT-II, we proceed from the $k_{\|}$ "moment" of the wave kinetic equation, which gives

$$
\begin{gathered}
\frac{\partial}{\partial t}\left\langle P_{\|}^{w}\right\rangle+\frac{1}{r} \frac{\partial}{\partial r}\left[r\left\langle\Pi_{r, \|}^{w}\right\rangle\right]-\int d \mathbf{k}\left\{\left\langle\frac{d k_{\|}}{d t} N\right\rangle+\frac{\partial k_{\|}}{\partial k_{r}}\left\langle\frac{d k_{r}}{d t} N\right\rangle\right\} \\
=\left\langle\int d \mathbf{k} k_{\|}\left(C_{w-w}(N)+C_{w-p}(N)\right)\right\rangle .
\end{gathered}
$$

Equation (17) describes the evolution of the mean parallel wave momentum $\left\langle P_{\|}^{w}\right\rangle$. Note that since the basic wave-wave interactions conserve momentum, we can immediately write

$$
\int d \mathbf{k} k_{\|} C_{w-w}(N)=0,
$$

i.e., wave-wave "collisions" conserve net wave momentum as a consequence of the $\mathbf{k}$ matching or selection rules. In practice, a concrete demonstration of Eq. (18) for a specific case requires writing $\int d \mathbf{k} k_{\|} C_{w-w}(N)$ as a double integral $\int d \mathbf{k} \int d \mathbf{k}^{\prime}$ symmetrizing $\mathbf{k}$ and $\mathbf{k}^{\prime}$, etc. Detailed examples of such calculations may be found in Ref. 54. The result of Eq. (18) still applies to the special case where one element of the wave triad is a zonal flow, though in that case the parallel 
momentum selection rule $k_{\|}=k_{\|}^{\prime}+k_{\|}^{\prime \prime}$ degenerates to the identity $k_{\|}=k_{\|}^{\prime}$, since zonal flows have essentially no variation along the field line. Stochastic straining by zonal flows conserves $N$, as well.

Since the wave-wave interaction process conserves momentum, the mean parallel wave momentum density equation may be simplified as

$$
\frac{\partial}{\partial t}\left\langle P_{\|}^{w}\right\rangle+\frac{1}{r} \frac{\partial}{\partial r}\left(r \Pi_{r, \|}^{w}\right)-\left\langle f_{\|}^{\mathrm{ref}}\right\rangle=\int d \mathbf{k}\left\langle k_{\|} C_{w-p}(N)\right\rangle .
$$

Here, we see that wave momentum density evolves due to the combined actions of the mean radial flux of parallel wave momentum $\left\langle\Pi_{r, \|}^{w}\right\rangle$, the parallel component of the mean refractive force (allowing for magnetic shear, i.e., $\partial k_{\|} / \partial k_{r} \neq 0$ )

$$
\left\langle f_{\|}^{\mathrm{ref}}\right\rangle=\int d \mathbf{k}\left\{\left\langle\frac{d k_{\|}}{d t} N\right\rangle+\frac{\partial k_{\|}}{\partial k_{r}}\left\langle\frac{d k_{r}}{d t} N\right\rangle\right\}
$$

and the net dissipation of mean wave momentum by all resonant wave-particle interactions, i.e.,

$$
\left(\frac{d\left\langle P_{\|}\right\rangle}{d t}\right)_{\text {diss }}=\int d \mathbf{k}\left\langle k_{\|} C_{w-p}(N)\right\rangle .
$$

However, since the wave momentum density dissipated by resonant interaction with the particles is simply the net rate of change of the resonant particle momentum density, we immediately have

$$
\begin{aligned}
\left(\frac{d\left\langle P_{\|}\right\rangle}{d t}\right)_{\text {diss }} & =\int d \mathbf{k}\left\langle k_{\|} C_{w-p}(N)\right\rangle \\
& =-\frac{d}{d t}\left\langle P_{\|}\right\rangle_{R}=-\frac{\partial}{\partial t}\left\langle P_{\|}\right\rangle_{R}-\frac{1}{r} \frac{\partial}{\partial r}\left[r\left\langle\widetilde{v}_{E r} \widetilde{P}_{\|}\right\rangle\right] .
\end{aligned}
$$

Note that the contribution from the divergence of the resonant particle momentum flux appears here because

it necessarily enters the time rate of change of the local resonant particle momentum density.

(ii) it is required for a treatment of wave-particle interactions that is consistent with that applied to the dissipation term.

Indeed, point (i) above follows from the fact that wave particle interaction in drift wave turbulence scatters particles in radius at the same time it accelerates particles along the field. Thus $C_{w-p}(N)$ and $\left\langle\widetilde{v}_{E r} \widetilde{P}_{\|}\right\rangle_{R}$ must be treated symmetrically and consistently, order by order in perturbation theory, as each is an element of the total wave particle interaction process. In principle, both $d\left\langle P_{\|}\right\rangle_{\text {diss }} / d t$ and $\left\langle\widetilde{v}_{E r} \widetilde{P}_{\|}\right\rangle_{R}$ contain contributions from all even orders in perturbation theory, and so may be written in the generic form ${ }^{55}$

$$
\begin{aligned}
\left(\begin{array}{c}
\left\langle\widetilde{v}_{E r} \widetilde{P}_{\|}\right\rangle \\
d\left\langle P_{\|}\right\rangle_{\mathrm{diss}}{ }^{\prime} d t
\end{array}\right)= & \sum_{n}\left\{\sum_{\mathbf{k}_{1}} \sum_{\mathbf{k}_{2}} \cdots \sum_{\mathbf{k}_{n}} C_{n}\left(\mathbf{k}_{1}, \mathbf{k}_{2}, \ldots, \mathbf{k}_{n}\right)\right. \\
& \times\left\langle\widetilde{\Phi}^{2}\right\rangle_{\mathbf{k}_{1}}\left\langle\widetilde{\Phi}^{2}\right\rangle_{\mathbf{k}_{2}} \cdots\left\langle\widetilde{\Phi}^{2}\right\rangle_{\mathbf{k}_{n}} \times \delta\left(\omega_{1}+\omega_{2}\right. \\
& \left.+\cdots+\omega_{n}-v_{\|[}\left[k_{1 \|}+k_{2 \|}+\cdots k_{n \|]}\right)\right\} .
\end{aligned}
$$

Here $n=1$ corresponds to the quasilinear piece, $n=2$ to the contribution from nonlinear Landau damping or Compton scattering, etc. Thus, the irreversibility underpinning of the $n=1$ contribution is stochasticity due to overlap of primary wave-particle resonances, that governing $n=2$ is stochasticity due to overlap of beat wave resonances, etc. Since here we are concerned only with the most basic mean field theory analysis, we limit $\Sigma_{n}$ to only the $n=1$ term and so, in the vein of quasilinear theory, retain only the primary resonance effects in the calculation of $\left\langle\widetilde{v}_{E r} \widetilde{P}_{\|}\right\rangle$.

Proceeding by combining Eqs. (19) and (20) and again utilizing Eq. (6c) to combine wave and resonant particle momentum finally leads us to the second or extended momentum conservation theorem (MT-II), which is

$$
\begin{aligned}
& \frac{d\left\langle\delta P_{\|}\right\rangle}{d t}+\frac{1}{r} \frac{\partial}{\partial r}\left[r\left\langle\widetilde{v}_{E r} \widetilde{P}_{\|}\right\rangle+r \int d \mathbf{k}\left\langle\left(v_{g r}+V_{r}\right) k_{\|} N\right\rangle\right] \\
& =\int d \mathbf{k}\left\{\left\langle\frac{d k_{\|}}{d t}\right\rangle\langle N\rangle+\frac{\partial k_{\|}}{\partial k_{r}}\left\langle\frac{d k_{r}}{d t}\right\rangle\langle N\rangle+\left\langle\left(\frac{d}{d t} \widetilde{k}_{\|}\right) \tilde{N}\right\rangle\right\} .
\end{aligned}
$$

Here we have written the mean refractive force as the product of the means plus the mean of the product of fluctuations. MT-II, as given by Eq. (22), states that the total parallel momentum density of the particles evolves by radial transport of resonant particle momentum, radial transport of parallel wave momentum due to magnetic shear (i.e., which renders $\left.\partial k_{\|} / \partial k_{r} \neq 0\right)$ and by the force exerted on the plasma due to refraction of waves [i.e. as given in the RHS of Eq. (22)]. Note that the refractive force density is a local momentum drive, and is not related to the divergence of a stress tensor, i.e., it represents a local force density exerted on the plasma, and has not been considered in previous studies. Clearly, MT-II is more general and inclusive than MT-I. Note also that if refractive force effects are ignored, the predictions of MT-I and MT-II are identical with regard to macroscopics (i.e., stresses).

At this point, it is appropriate to discuss the physics of the refractive force in some depth. As mentioned above, the refractive force is a simple and straightforward consequence of the fact that bending of wave ray trajectories in a dielectric medium (e.g., plasma) necessarily induces a recoil force on that medium. The refractive force is not a priori dependent upon dissipation of wave energy, wave breaking, nonlinear transfer or any other "strongly nonlinear" processes. Note that the refractive force consists of the sum of two pieces, a mean or coherent piece $\left(\sim\left\langle d k_{\|} / d t\right\rangle\langle N\rangle+\left(\partial k_{\|} / \partial k_{r}\right)\right.$ $\left.\times\left\langle d k_{r} / d t\right\rangle\langle N\rangle\right)$ and a stochastic piece $\left[\sim\left\langle\left(d \widetilde{k}_{\|} / d t\right) \tilde{N}\right\rangle\right]$. The coherent refractive force $f_{\|, \text {coh }}^{\text {ref }}$ is given by 


$$
f_{\|, \mathrm{coh}}^{\mathrm{ref}}=-\int d \mathbf{k}\left\{\left\langle\nabla_{\|}(\omega+\mathbf{k} \cdot \mathbf{V})\right\rangle\langle N\rangle+\frac{\partial k_{\|}}{\partial k_{r}} k_{\theta}\left\langle v_{E}\right\rangle^{\prime}\langle N\rangle\right\} .
$$

In practice the first term will occur as a result of poloidal asymmetry in mean plasma flows or the parameters which determine the mean wave frequency, whereas the second term will be relevant in the presence of poloidal flow shear in sheared magnetic geometry. Also note that for the second term, since $\partial k_{\|} / \partial k_{r} \sim k_{\theta}$, the overall term $\sim k_{\theta}^{2}$, so no additional poloidal symmetry breaking is required. The stochastic component of the refractive force may be calculated using a quasilinear approximation to the mean product $\left\langle\left(d \widetilde{k}_{\|} / d t\right) \tilde{N}\right\rangle$ and the linear response for $\tilde{N}$. Thus,

$$
\tilde{N}_{\mathbf{q}, \Omega}=-R\left(\Omega-\mathbf{q} \cdot \mathbf{v}_{g}\right)(\widetilde{\omega}+\mathbf{k} \cdot \widetilde{\mathbf{V}})_{\mathbf{q}} \mathbf{q} \cdot \frac{\partial\langle N\rangle}{\partial \mathbf{k}},
$$

where

$$
R\left(\Omega-\mathbf{q} \cdot \mathbf{v}_{g}\right)=\frac{\left|\gamma_{\mathbf{k}}\right|}{\left(\Omega-\mathbf{q} \cdot \mathbf{v}_{g}\right)^{2}+\left|\gamma_{\mathbf{k}}\right|^{2}}
$$

is the strain field wave packet resonance function. Here $\mathbf{q}$ and $\Omega$ are the wave vector and frequency of the strain field, respectively. Thus

$$
f_{\|, \text {stoch }}^{\mathrm{ref}}=-\hat{\mathbf{b}} \cdot \overleftrightarrow{D_{\mathbf{k}}} \cdot \frac{\partial\langle N\rangle}{\partial \mathbf{k}},
$$

where $\overleftrightarrow{D_{\mathbf{k}}}$ is the $\mathbf{k}$-space diffusion tensor,

$$
\overleftrightarrow{D_{\mathbf{k}}}=\sum_{\mathbf{q}} \mathbf{q q}\left|(\widetilde{\omega}+\mathbf{k} \cdot \widetilde{\mathbf{V}})_{\mathbf{q}}\right|^{2} R\left(\Omega-\mathbf{q} \cdot \mathbf{v}_{g}\right)
$$

and $\hat{\mathbf{b}} \cdot \overleftrightarrow{D_{\mathbf{k}}}$ is given by

$$
\hat{\mathbf{b}} \cdot \overleftrightarrow{D_{\mathbf{k}}}=\sum_{\mathbf{q}} q_{\|} \mathbf{q}\left|(\widetilde{\omega}+\mathbf{k} \cdot \tilde{\mathbf{V}})_{\mathbf{q}}\right|^{2} R\left(\Omega-\mathbf{q} \cdot \mathbf{v}_{g}\right)
$$

Equation (23e) indicates that the correlations between $q_{\|}$and the other components of the strain field wave vector are critical to determining various components of the net refractive force and that the parallel (i.e., poloidal) variation of the strained field is the ultimate origin of the parallel stochastic refractive force. This observation immediately suggests that geodesic acoustic modes (GAMs) are natural candidates for a physical origin of the refractive force, since on account of their poloidal variation, they produce a strain field with nonzero $q_{\|}$and $q_{\theta}$. All these considerations suggest a simpler and more explicit version of (MT-II), which is just

$$
\begin{aligned}
\frac{d\left\langle\delta P_{\|}\right\rangle}{d t}+ & \frac{1}{r} \frac{\partial}{\partial r}\left[r\left\langle\widetilde{v}_{E r} \widetilde{P}_{\|}\right\rangle+r \int d \mathbf{k}\left\langle\left(v_{g r}+V_{r}\right) k_{\|} N\right\rangle\right] \\
= & -\int d \mathbf{k}\left\{\nabla_{\|}\langle\omega+\mathbf{k} \cdot \mathbf{V}\rangle\langle N\rangle+\frac{\partial k_{\|}}{\partial k_{r}} k_{\theta}\left\langle v_{E}\right\rangle^{\prime}\langle N\rangle\right. \\
& \left.+\hat{\mathbf{b}} \cdot \overleftrightarrow{D_{\mathbf{k}}} \cdot \frac{\partial\langle N\rangle}{\partial \mathbf{k}}\right\} .
\end{aligned}
$$

We now discuss the general structure of the momentum balance relation and its implications for intrinsic rotation. More generally, to address the phenomenon of intrinsic rotation demands an affirmative answer to the question of "can the turbulence sustain a stationary, nontrivial (i.e., peaked) profile of toroidal rotation in the absence of external sources?" For intrinsic rotation at an arbitrary radius $a$, this requires that a nontrivial profile result from the stationary solution to Eq. (24), within $r=a$. Imposing the standard boundary condition that fluxes vanish on axis then leads to the momentum stationarity condition,

$$
\begin{aligned}
& \left.\left(r\left\langle\widetilde{v}_{E r} \widetilde{P}_{\|}\right\rangle_{R}+r\left\langle\Pi_{r, \|}^{w}\right\rangle\right)\right|_{a} \\
& =-\int_{0}^{a} r^{\prime} d r^{\prime} \int d \mathbf{k}\left[\left\langle\nabla_{\|}(\omega+\mathbf{k} \cdot \mathbf{V})\right\rangle\langle N\rangle\right. \\
& \left.\quad+\frac{\partial k_{\|}}{\partial k_{r}} k_{\theta}\left\langle v_{E}\right\rangle^{\prime}\langle N\rangle+\hat{\mathbf{b}} \cdot \overleftrightarrow{D_{\mathbf{k}}} \cdot \frac{\partial\langle N\rangle}{\partial \mathbf{k}}\right],
\end{aligned}
$$

i.e., that the resonant particle momentum flux at $r=a$ plus the wave momentum flux at $r=a$ balance the refractive force at $r=a$. The resonant particle momentum flux is calculated in detail in Sec. IV. We can, however, expect it to have a structure of the general form,

$$
\left\langle\widetilde{v}_{E r} \widetilde{P}_{\|}\right\rangle_{R}=-D_{\|} \frac{\partial}{\partial r}\left\langle P_{\|}\right\rangle+\left(\Pi_{r, \|}\right)_{R}^{\mathrm{ND}},
$$

where the nondiffusive piece due to resonant particles $\left(\Pi_{r, \|}\right)_{R}^{\mathrm{ND}}$ may be further decomposed into convective and residual stress components, i.e.,

$$
\left(\Pi_{r, \|}\right)_{R}^{\mathrm{ND}}=V_{r}\left\langle P_{\|}\right\rangle+\left(\Pi_{r, \|}\right)_{R}^{\text {residual }} .
$$

Note that further information or assumptions concerning the specific dynamical model are required to separate the particle flux and flow transport (stress) contributions to $\left(\Pi_{r, \|}\right)_{R}^{\mathrm{ND}}$. In general, however, sustaining a nontrivial (positive) flow profile requires at least one of

$$
\left(\Pi_{r, \|}\right)_{R}^{\mathrm{ND}}<0,
$$

i.e., either inward resonant convection of momentum $\left(V_{r}<0\right)$ or an inward resonant residual stress $\left(\Pi_{r, \|}\right)_{R}^{\text {residual }}$ $<0$, or

$$
\left(\Pi_{r, \|}\right)_{R}^{w}<0
$$

i.e., either an inflow of positive, or an outflow of negative, wave momentum, or

$$
\int_{0}^{a} f_{\text {ref }} d r \neq 0
$$

i.e., a net refractive force.

It is interesting to note that the microscopic irreversibility which underpins $\left\langle\widetilde{v}_{E r} \widetilde{P}_{\|}\right\rangle$is stochasticity of resonant particles, while that which underlies $\left(\Pi_{r, \|}\right)_{R}^{w}$ is an overlap of wave resonances or drift wave ray chaos resulting from interaction with zonal flow or GAM shears. Ray chaos is also the underpinning of the stochastic refractive force.

Further progress clearly requires concrete calculation of $\left\langle\Pi_{r, \|}^{w}\right\rangle$, the mean wave momentum flux, and of $\left\langle\widetilde{v}_{E r} \widetilde{P}_{\|}\right\rangle$, the resonant particle momentum flux. Loosely speaking, one can expect the former to be more important for fluid-like instabilities and/or in regimes of strong turbulence, while the latter to be more important in kinetic regimes near marginality, 
i.e., for stiff profiles. We expect the refractive force to be significant only when poloidal symmetry is strongly broken, or when electric field shear is strong. In practice, this is likely to occur only at the plasma edge or in transport barriers. Given the special nature and complexity of the edge plasma transport and barrier dynamics, we postpone further discussion of the effects of the refractive force on rotation to a future paper.

\section{CALCULATING THE WAVE MOMENTUM FLUX: A CHAPMAN-ENSKOG APPROACH}

In this section, we present the concrete calculation of $\left\langle\Pi_{r, \|}^{w}\right\rangle$, the mean radial flux of parallel wave momentum. This is one of the two principal components of the total momentum flux, and is most important in regimes of strong, hydrodynamic-like turbulence.

The key physical idea which enables us to systematically calculate $\left\langle\Pi_{r, \|}^{w}\right\rangle=\int d \mathbf{k}\left\langle v_{g r} k_{\|} N\right\rangle$ is that waves propagate while being scattered by nonlinear interaction. Hence we develop an approach similar to that used to describe quasiparticle transport processes in solids and Fermi liquids. ${ }^{56}$ Hence, it is useful to construct a kinetic theory of the wave population density $N$ in the phase space of $\mathbf{k}$ and $\mathbf{x}$. To this end, we first list and order the basic time and space scales. Drift wave packets propagate radially at $v_{\mathrm{gr}}=-2 k_{\theta} k_{r} \rho_{s}^{2} v * /\left(1+k_{\perp}^{2} \rho_{s}^{2}\right)^{2}$, where $v *=\rho_{s} c_{s} / L_{n}$ is the diamagnetic velocity. Thus a drift wave packet will:

Propagate one radial wavelength in

$$
\left(\tau_{\text {prop }}\right)_{\mathbf{k}} \sim\left(k_{r} v_{\mathrm{gr}}\right)^{-1} \sim-\frac{\left(1+k_{\perp}^{2} \rho_{s}^{2}\right)^{2}}{2 k_{\theta} k_{r}^{2} \rho_{s}^{2} v *} \geq \omega_{\mathbf{k}}^{-1} .
$$

(ii) Scatter or decay via nonlinear interaction in

$$
\left(\tau_{\text {decay }}\right)_{\mathbf{k}} \sim\left(k_{\perp}^{2} D\right)^{-1} \sim \omega_{\mathbf{k}}^{-1},
$$

where the equalities follow from using the "mixing length" prediction of fluctuation levels, but should be regarded as crude estimates, only. Zonal flow shearing will yield a roughly comparable decay time. It is useful to note that where fluctuation levels are comparable to the mixing-length level, $\left(\tau_{\text {prop }}\right)_{\mathbf{k}} \sim\left(\tau_{\text {decay }}\right)_{\mathbf{k}}$ follows directly from $\widetilde{v} \sim v *$. For smaller fluctuation levels, i.e., $(\widetilde{v}<v *)$, then $\left(\tau_{\text {prop }}\right)_{\mathbf{k}} \ll\left(\tau_{\text {decay }}\right)_{\mathbf{k}}$, so wave transport is the faster process.

(iii) Propagate one fluctuation intensity gradient scale length distance $\left(L_{I}\right.$, where $\left.L_{I}^{-1} \equiv\langle N\rangle^{-1} \partial\langle N\rangle / \partial r\right)$ in

$$
\left(\tau_{\text {prop }}\right)_{L_{I}} \sim\left(k_{r} L_{I}\right)\left(\tau_{\text {prop }}\right)_{\mathbf{k}} .
$$

These time scales are summarized in Table I. Hence, for the "typical" regime where $k_{r} L_{I} \gg 1$, we are justified in applying the ordering

$$
\left(\tau_{\text {prop }}\right)_{\mathbf{k}} \sim\left(\tau_{\text {decay }}\right)_{\mathbf{k}} \ll\left(\tau_{\text {prop }}\right)_{L_{I}} .
$$

This implies that a drift wave packet will be nonlinearly scattered much more rapidly than it will transit one intensity profile scale length. Thus, drift wave packets can be thought of as having "short mean free path" or, in the language of radiation hydrodynamics, as satisfying the conditions for a
TABLE I. Time scales for the wave population response.

\begin{tabular}{lc}
\hline \hline Time scale & Definition \\
\hline Oscillation time & $\tau \sim \omega_{\mathbf{k}}^{-1}$ \\
Self-propagation (time to propagate $\left.\lambda_{r}\right)$ & $\left(\tau_{\text {prop }}\right)_{\mathbf{k}} \sim\left(k_{r} v_{\mathrm{gr}}\right)^{-1}$ \\
Decay time (time of nonlinear scattering) & $\tau_{\text {decay }} \sim\left(k_{\perp}^{2} D\right)^{-1}$ \\
Intensity gradient scale propagation time & $\left(\tau_{\text {prop }}\right)_{L_{I}} \sim L_{I} / v_{\mathrm{gr}}$ \\
& $\sim\left(k_{r} L_{I}\right) \tau_{\text {prop }} \mathbf{k}$ \\
\hline \hline
\end{tabular}

regime of large optical depth. ${ }^{33}$ Central to this ordering is the assumption of a clear separation of scales between the radial wavelength $\left(k_{r}^{-1}\right)$ and/or correlation length $\Delta r_{c}$ on the one hand, and the intensity profile scale length $L_{I}$ on the other. $L_{I}$ may be loosely estimated as $L_{\perp}$ (i.e., a typical profile scale length), since the turbulence intensity profile is supported by the heat and particle fluxes which produce the profiles of plasma temperature, density, etc. Alternatively put, since $T(r)$ decays with radius for central deposition, $\langle N(r)\rangle$ must increase with radius (see Fig. 1). This is both independently plausible and also consistent with the empirical finding that the fluctuation intensity profile is almost always observed to increase with radius. ${ }^{57}$ One possible exception to the assumed $L_{I} k_{r} \gg 1$ ordering would be the instance of a fully developed strong transport barrier.

Given time and space scale ordering discussed above, we can formulate a Chapman-Enskog expansion approach to the calculation of $\Pi_{r, \| \cdot}^{w}$. The basic idea is to write

$$
N=\langle N(\mathbf{x}, \mathbf{k}, t)\rangle+\delta N,
$$

where $\langle N\rangle$ is the slowly varying "mean" wave population density, and $\delta N$ is the perturbation to it induced by gradients of $\langle N\rangle$ in radius and $\mathbf{k}$. Since, $\delta N$ will be shown to be proportional to $\partial\langle N\rangle / \partial r$ and $\partial\langle N\rangle / \partial k_{r}$, it ultimately produces the net flux of wave momentum. As usual, $\delta N$ is calculated perturbatively, exploiting the time scale ordering.

Proceeding, we hereafter ignore the mean radial convection, and write the wave kinetic equation in the form,

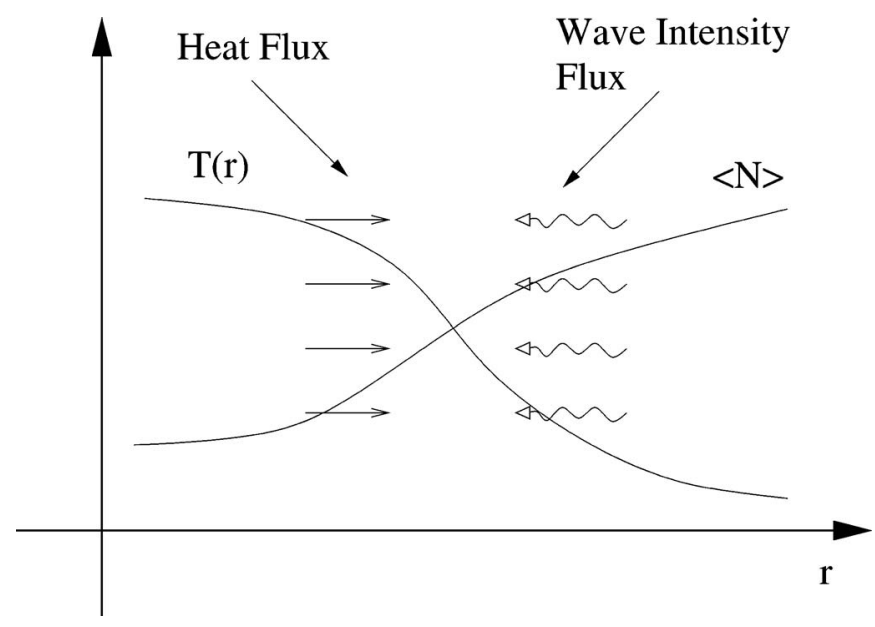

FIG. 1. Cartoon of radial profile of temperature and turbulence intensity. Solid arrows denote outward flux of heat, whereas the squiggles indicate an inward flux of wave intensity. 


$$
\frac{\partial N}{\partial t}+\mathbf{v}_{g} \cdot \nabla N-\frac{\partial}{\partial \mathbf{x}}(\mathbf{k} \cdot \mathbf{V}) \frac{\partial}{\partial \mathbf{k}} N=C(N),
$$

where

$$
N=\langle N\rangle+\delta N .
$$

We assume the "collisions" (i.e., the nonlinear wave-wave and wave-particle scattering events) underpinning $C(N)$ occur with collision frequency $\nu_{T \mathbf{k}} \sim \Delta \omega_{\mathbf{k}} \sim 1 /\left(\tau_{\text {decay }}\right)_{\mathbf{k}}$ (where $\Delta \omega_{\mathbf{k}}$ is the decorrelation rate for scale $\mathbf{k}$ ), and that $v_{\mathrm{gr}} / L_{I} \ll \Delta \omega_{\mathbf{k}}$. More precisely, $\nu_{T \mathbf{k}}$ is the characteristic rate of wave-wave energy transfer or wave particle scattering, as represented by $C(N)$. Here $C(N)$ includes both $C_{w-w}$ and $C_{w-p}$ and all relevant orders (i.e., both linear and nonlinear) of each type of scattering process. Thus, to lowest order, Eq. (28a) is just

$$
C(N)=0
$$

which formally defines the mean $\langle N\rangle$ as

$$
N^{(0)}=\langle N\rangle \text {. }
$$

The restrictions and caveats concerning the structure of $\langle N\rangle$ require a brief discussion. In practice, the mean $\langle N\rangle$ is defined by the balance of linear growth with coupling to dissipation via nonlinear wave-wave and wave-particle interaction. Here "wave-wave interaction" includes the possibility of zonal shearing, and $\nu_{T}$ is the characteristic rate for nonlinear relaxation to this mean state. Obviously, $\nu_{T \mathbf{k}} \sim \Delta \omega_{\mathbf{k}}$, the turbulence decorrelation rate. In general, we can expect $\langle N\rangle$ to have a power law structure, in between limits set by low$k$ (i.e., flow damping) and high- $k$ (i.e., Landau damping and collisions) dissipation. Thus, there may be some limitation on which higher order moments of $\langle N\rangle$ actually exist without sensitive dependence upon dissipation. We defer detailed discussion of this subtle question to a future publication. $\langle N\rangle$ must be symmetric in $k_{r}$, since there should be no intrinsically preferred direction of wave packet propagation or the condition of stationarity of $\langle N\rangle$ would be violated. Thus, $\langle N\rangle$ makes no contribution to $\left\langle\Pi_{r, \|}^{w}\right\rangle$. Since $N=\langle N\rangle+\delta N$, to next order we have

$$
\begin{aligned}
\frac{\partial}{\partial t} \delta N & +\mathbf{v}_{g} \cdot \nabla \delta N-\frac{\partial}{\partial r}\left(k_{\theta}\left\langle v_{E}\right\rangle\right) \frac{\partial}{\partial k_{r}} \delta N-\nu_{T} \delta N \\
& =-v_{\mathrm{gr}} \frac{\partial\langle N\rangle}{\partial r}+\frac{\partial}{\partial r}\left(k_{\theta}\left\langle v_{E}\right\rangle\right) \frac{\partial\langle N\rangle}{\partial k_{r}}
\end{aligned}
$$

with $k_{\theta} \rightarrow m / r$, where $m$ is the poloidal mode number. Here we have assumed that the mean flow is predominantly a sheared $E \times B$ poloidal flow, so

$$
\mathbf{k} \cdot \mathbf{V}=k_{\theta}\left\langle v_{E}\right\rangle \text {. }
$$

Also, we have employed a Krook approximation to $C(N)$, i.e.,

$$
C\left(N_{\mathbf{k}}\right) \cong-\nu_{T \mathbf{k}}\left(N_{\mathbf{k}}-\langle N\rangle\right)=-\nu_{T \mathbf{k}} \delta N_{\mathbf{k}} .
$$

This states that the effect of wave-wave or wave-particle collisions on $N$ is simply to drive it to relax to the mean $\langle N\rangle$. Recall that the Manley-Rowe relations state that $C(N)$ does not need to conserve the quanta number, so a Krook model will suffice. Then, in the limit of strong collisionality, where $\nu_{T}>v_{\mathrm{gr}} / L_{I}, \Omega, k_{\theta}\left\langle v_{E}\right\rangle^{\prime} / k_{r}$, etc., we have

$$
\delta N=-\frac{v_{\mathrm{gr}}}{\nu_{T_{\mathbf{k}}}} \frac{\partial\langle N\rangle}{\partial r}+\frac{1}{\nu_{T_{\mathbf{k}}}} \frac{\partial}{\partial r}\left(k_{\theta}\left\langle v_{E}\right\rangle\right) \frac{\partial\langle N\rangle}{\partial k_{r}} .
$$

Here $\delta N$, the excursion from the uniform "equilibrium" distribution is driven by both $\partial\langle N\rangle / \partial r$ and $\partial\langle N\rangle / \partial k_{r}$, i.e., the quanta density profile gradients in both radius and radial wave-number. Substituting $\delta N$ from Eq. (31) into $\left\langle\Pi_{r, \|}^{w}\right\rangle$ then gives

$$
\begin{aligned}
\left\langle\Pi_{r, \|}^{w}\right\rangle & =\int d \mathbf{k} v_{\mathrm{gr}} k_{\|}\left\{-\frac{v_{\mathrm{gr}}}{\nu_{T_{\mathbf{k}}}} \frac{\partial\langle N\rangle}{\partial r}+\frac{k_{\theta}}{\nu_{T_{\mathbf{k}}}} \frac{\partial\left\langle v_{E}\right\rangle}{\partial r} \frac{\partial\langle N\rangle}{\partial k_{r}}\right\} \\
& =-\int d \mathbf{k} k_{\|}\left\{\frac{v_{\mathrm{gr}}^{2}}{\nu_{T_{\mathbf{k}}}} \frac{\partial\langle N\rangle}{\partial r}+\frac{\partial}{\partial k_{r}}\left(\frac{v_{\mathrm{gr}}}{\nu_{T_{\mathbf{k}}}}\right)\left\langle v_{E}\right\rangle^{\prime} k_{\theta}\langle N\rangle\right\},
\end{aligned}
$$

where we have integrated by parts to rewrite the second term on the RHS. It is apparent that the wave momentum flux consists of two pieces. The first is a Fickian flux, driven by $\partial\langle N\rangle / \partial r$ with effective diffusivity $D_{\text {rad }} \sim v_{\mathrm{gr}}^{2} / \nu_{T \mathbf{k}}$. The second is a flow shear driven "mobility," proportional to the divergence in $k_{r}$ of the wave packet group velocity. The second term arises from the combined effects of shearing, which tends to increase $k_{r}$ by straining, and the presence of a population density profile in $k_{r}$, i.e., $\partial\langle N\rangle / \partial k_{r}$. Thus $\left\langle\Pi_{r, \|}^{w}\right\rangle$ may be written in the approximate diffusion-convection form,

$$
\left\langle\Pi_{r, \|}^{w}\right\rangle=-D_{w} \frac{\partial}{\partial r}\left\langle P_{\|}^{w}\right\rangle+V_{w}\left\langle P_{\|}^{w}\right\rangle,
$$

where the wave momentum diffusivity is

$$
D_{w} \cong \frac{\int d \mathbf{k}\left(\frac{v_{\mathrm{gr}}^{2}}{\nu_{T_{\mathbf{k}}}}\right)\langle N\rangle}{\int d \mathbf{k}\langle N\rangle}
$$

and the convection velocity is

$$
V_{w}=-\frac{\int d \mathbf{k}\left[\frac{\partial}{\partial k_{r}}\left(\frac{v_{\mathrm{gr}}}{\nu_{T_{\mathbf{k}}}}\right) k_{\theta}\left\langle v_{E}\right\rangle^{\prime}\right]\langle N\rangle}{\int d \mathbf{k}\langle N\rangle} .
$$

Of course, the first term on the RHS of Eq. (32a) gives the diffusive flux, while the second term drives the convective flux. Given the identity between wave-momentum density and nonresonant particle momentum density, $\left\langle\Pi_{r, \|}^{w}\right\rangle$ directly implies a corresponding flux of nonresonant particle momentum. However since the convention is to define transport coefficients such as diffusion, convection, etc. in terms of how they act on moments of the particle distribution function, $\left\langle\Pi_{r, \|}^{w}\right\rangle$ ultimately is classified as a contribution to the residual stress part of the radial flux of parallel momentum. Finally, we note that, at this stage of the calculation the origin of symmetry breaking (i.e., the origin of $\left.\left\langle k_{\|}\right\rangle=\int d \mathbf{k} k_{\|}\langle N\rangle / \int d \mathbf{k}\langle N\rangle \neq 0\right)$ has not yet been addressed. 


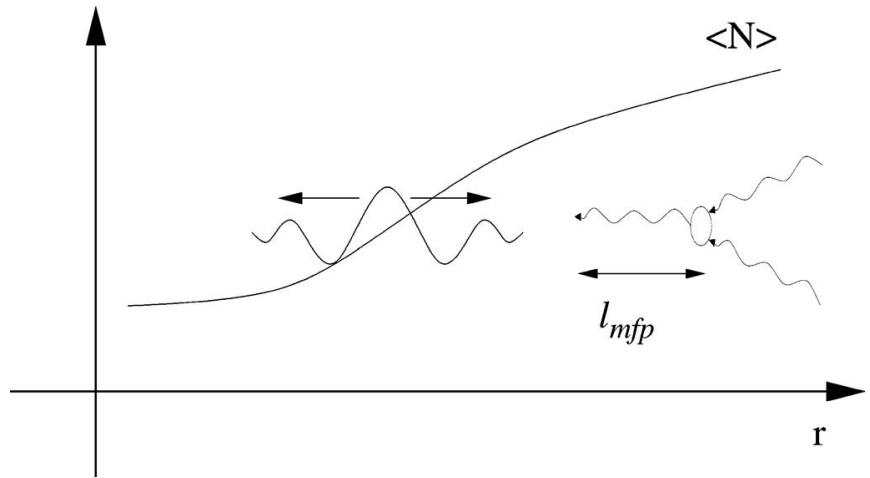

FIG. 2. Cartoon of radial profile of turbulence intensity. Wave propagation direction is equally likely. However, inhomogeneity in turbulence intensity provides a means of net flux of wave intensity. Here we consider regimes in which $l_{\mathrm{mfp}}$ is small in comparison to the profile variation.

The physics of the first $\partial\langle N\rangle / \partial r$-driven contribution to $\left\langle\Pi_{r, \|}^{w}\right\rangle$ is straightforward, and describes diffusion of wave momentum due to propagation and scattering of the wave packets which carry the momentum. ${ }^{33}$ As shown in Fig. 2, since $v_{\mathrm{gr}} \sim k_{r}$ (as $\chi \sim-k_{\perp}^{2} \rho_{s}^{2}$ for drift waves), a wave packet originating at a particular radius $r$ will propagate a distance $\ell_{\mathrm{mfp}} \sim v_{\mathrm{gr}} \tau_{c}$ before undergoing absorption and re-emission as part of the nonlinear wave-wave interaction process. This immediately implies the existence of a diffusive flux of wave momentum $\left\langle\Pi_{r, \|}^{w}\right\rangle_{D}=-D_{\mathrm{rad}} \partial\left\langle P_{\|}\right\rangle / \partial r \quad$ with $\quad D_{\mathrm{rad}} \sim v_{\mathrm{gr}} \ell_{\mathrm{mfp}}$ $\sim v_{\mathrm{gr}}^{2} / \nu_{T}$. It is important to realize that this flux is due to wave radiation (albeit in the short mean free path limit) rather than turbulent mixing. Interestingly, since, for "typical" mixing length type fluctuation levels, $v_{\mathrm{gr}} \sim v *, \nu_{T} \sim \omega_{\mathbf{k}}$ $\sim c_{S} / L_{\perp}$, we have $D_{\mathrm{rad}} \sim D_{\mathrm{GB}}$ so that the radiative diffusivity $D_{\text {rad }}$ is similar to the familiar gyro-Bohm turbulent diffusivity! This, together with the presence of a heat flux driving and the fact that $n T \gg \varepsilon_{\omega}$, explains why the edge peaked intensity gradient does not simply collapse via wave radiation. It also explains the long-standing difficulty in disentangling wave transport processes ${ }^{38}$ from eddy transport processes, since transport of both heat and wave intensity will occur at a similar rate. One interesting consequence of this wave quanta diffusion process is that since the turbulent intensity field is always peaked toward the edge, there will always be a concomitant inward diffusive flux of wave quanta. This implies a spreading of edge turbulence inward into the core by radiative diffusion, as originally predicted by Kadomtsev. ${ }^{58}$ Though parallel momentum density and wave momentum density are, of course, different moments of $\langle N\rangle$, a similar inward diffusive flux of wave momentum from the edge can also be expected. Indeed, given that the parallel wave momentum flux corresponds to a portion of the parallel Reynolds stress $\left\langle\widetilde{v}_{r} \widetilde{v}_{\|}\right\rangle$and that stress induced change in momentum necessarily involves an intensity gradient, it is no surprise that $\left\langle\Pi_{r, \|}^{w}\right\rangle$ contains an intensity gradient dependent contribution. This is significant since several symmetry breaking mechanisms may be at work in the edge plasma, ${ }^{15}$ so such inward quanta diffusion would transmit the broken symmetry, as well.

The physics of the second, $\partial\langle N\rangle / \partial k_{r}$ driven contribution

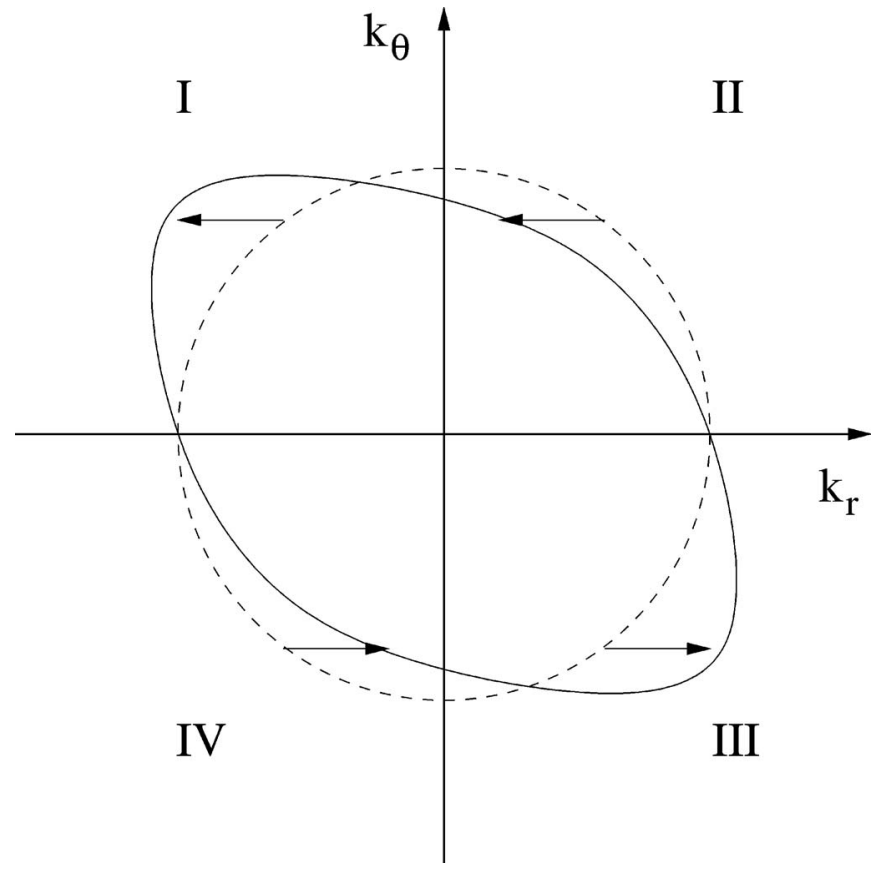

FIG. 3. Contours of $N_{k}$ in the $k_{\theta}-k_{r}$ plane. The broken line corresponds to an initial isotropic case, the solid line to the spectrum after being refracted by mean shear flow. It is apparent that the wave population in regions I and III increases while regions II and IV decrease.

to $\left\langle\Pi_{r, \|}^{w}\right\rangle$ (from which the convective wave momentum flux originates) is due to the distortion in $N$ induced by shearing, which tends to increase $k_{r}$. Formally, $\delta N \sim \tau_{c \mathbf{k}}\left(\partial k_{r} / \partial t\right)$ $\times\left(\partial\langle N\rangle / \partial k_{r}\right) \sim \tau_{c \mathbf{k}} k_{\theta}\left\langle v_{E}\right\rangle^{\prime} \partial\langle N\rangle / \partial k_{r}$, so $\left\langle\Pi_{r, \|}^{w}\right\rangle \sim \int d \mathbf{k} k_{\|} v_{\mathrm{gr}} \delta N$ is just the flux of wave momentum which occurs during the change in population-density induced by a shearing event. The key element here is $k_{r}$ dependence, i.e., shearing alters $k_{r}$, and since both $v_{\mathrm{gr}}$ and $\langle N\rangle$ are functions of $k_{r}$, a flux of wave momentum density results. Thus, one way to understand this convective momentum flux is as a process of wave "wind-up" due to shear, as shown in Fig. 3. Note, however that this effect is conceptually distinct from its better known counterpart related to wind up of $k_{\|}$with $k_{r}$, which we discuss later in the context of symmetry breaking. Alternatively, an integration by parts gives

$$
\left\langle\Pi_{r, \|}^{w}\right\rangle_{\text {convective }} \cong \int d \mathbf{k}\left[\frac{\partial}{\partial k_{r}}\left(\frac{v_{\mathrm{gr}}}{\nu_{T_{\mathbf{k}}}}\right)\right] k_{\theta}\left\langle v_{E}\right\rangle^{\prime}\langle N\rangle,
$$

thus linking the convective flux to the $k_{r}$ dependence of $v_{\mathrm{gr}}$. This suggests that when shearing alters $k_{r}$, the variation in wave packet propagation speed (i.e., the "compressibility" of $v_{\mathrm{gr}}$ with respect to $k_{r}$ ) will then induce a net wave momentum flux. Note that since $v_{\mathrm{gr}} \sim k_{\theta},\left\langle\Pi_{r, \|}^{w}\right\rangle_{\text {convective }}$ is even in $k_{\theta}$, so no additional poloidal symmetry breaking (i.e., other than that driven by $\left\langle v_{E}\right\rangle^{\prime}$ ) is required in order to induce a flux. Finally, it is useful to observe that since $v_{\mathrm{gr}} \sim v^{*},\left\langle\Pi_{r, \|}^{w}\right\rangle$ $\sim v *\left\langle v_{E}\right\rangle^{\prime}$, so the off-diagonal wave-momentum flux is determined by the product of the electric field shear and the mode propagation velocity $\left(v *_{e}\right.$ or $\left.v *_{i}\right)$. Thus a sudden change or bifurcation in the convective momentum flux can be induced by either 
An abrupt change in $\left\langle v_{E}\right\rangle^{\prime}$, as in an electric field shear induced transport bifurcation, as observed for example in L-H mode transition and ITB formation.

(ii) A change in mode propagation direction, such as would occur along with a change in the mode population composition. One example of this might be a change from predominance of $v *_{e}$-direction propagating waves (i.e., electron drift waves) to $v *_{i}$-direction propagating waves (i.e., ion drift waves or ITG modes) as the density $n$ exceeds the threshold for excitation of ITG modes at saturation of energy confinement in $\mathrm{OH}$ plasmas.

Note that momentum transport bifurcations originating with a change in $V_{w}$ thus can occur either in connection with a change in confinement (i.e., $\left\langle v_{E}\right\rangle^{\prime}$ increases, thus suppressing turbulence and forming a transport barrier) or as a change in momentum transport at roughly constant thermal energy content. We also comment that shearing and wave refraction can also induce a convective flux of $\langle N\rangle$, as well as $\left\langle P_{\|}\right\rangle$, and so may play a role in the dynamics of turbulence spreading. Finally, we note that the total momentum flux should include the contribution from convection by radial flows (i.e., due to streamers, for example) and so is

$$
\begin{aligned}
\left\langle\Pi_{r, \|}^{w}\right\rangle= & \int d \mathbf{k} k_{\|}\left\{\left\langle V_{r}\right\rangle\langle N\rangle-\frac{v_{\mathrm{gr}}^{2}}{\nu_{T_{\mathbf{k}}}} \frac{\partial\langle N\rangle}{\partial r}\right. \\
& \left.+v_{\mathrm{gr}} \frac{k_{\theta}}{v_{T_{\mathbf{k}}}}\left\langle v_{E}\right\rangle^{\prime} \frac{\partial\langle N\rangle}{\partial k_{r}}\right\} .
\end{aligned}
$$

All the results given up until now have been calculated for the regime of strong scattering, where $\Omega, v_{\mathrm{gr}} / L_{I}$, $k_{\theta}\left\langle v_{E}\right\rangle^{\prime} / k_{r} \ll \nu_{T_{\mathbf{k}}}$. However, in regimes of weaker turbulence and/or sharper intensity or electric field gradients, the inequality above may break down, so that $\ell_{\mathrm{mfp}}>L_{I}$, etc. This forces us to confront the analog of flux limited radiative transfer. In that case, and considering the spatial flux, for example, the stationary, gradient driven perturbation would follow from

$$
\left(\mathbf{v}_{g} \cdot \nabla+\nu_{T_{\mathbf{k}}}\right) \delta N=-v_{\mathrm{gr}} \frac{\partial\langle N\rangle}{\partial r}
$$

so the response $\delta N$ would no longer be local, but instead take the integral form

$$
\delta N=-\int_{r_{a}}^{r} d r^{\prime} K\left(r, r^{\prime}, t\right) v_{\mathrm{gr}} \frac{\partial\left\langle N\left(r^{\prime}\right)\right\rangle}{\partial r},
$$

where $K\left(r, r^{\prime}, t\right)$ is a nonlocal influence kernel of width $\ell_{\mathrm{mfp}}$, which is obtained by inverting the LHS of Eq. (34a) ${ }^{59}$ Continuing in this vein, a crude approximation would eventually yield a tractable form for $D_{\text {rad }}$, such as

$$
D_{\mathrm{rad}} \cong v_{\mathrm{gr}}^{2}\left[\frac{\nu_{T}}{\left(v_{\mathrm{gr}} / L_{I}\right)^{2}+\nu_{T}^{2}}\right] .
$$

Thus for smooth intensity profiles and strong scattering where $\nu_{T}>v_{\mathrm{gr}} / L_{I}$, etc., $D_{\mathrm{rad}} \sim v_{\mathrm{gr}}^{2} / \nu_{T}$, as before. On the other hand, for steep profiles and/or weak scattering (with consequent long $\ell_{\mathrm{mfp}}$ ),

$$
D_{\mathrm{rad}} \cong \frac{v_{\mathrm{gr}}^{2} \nu_{T}}{\left(v_{\mathrm{gr}} / L_{I}\right)^{2}} \cong \nu_{T} L_{I}^{2},
$$

as in the case of flux limited transport. ${ }^{33}$ Similar approximate forms which ensure proper behavior in the regimes of long packet mean free path, weak scattering, strong shearing or fast variation can easily be derived. In general, in such cases the response is nonlocal in space and/or time, so $\delta N$ is given by a response integral. In the limit of very long packet mean free path, a coherent wave-packet dynamics approach, using integration along rays (in the spirit of Longuett-Higgins and Stewart, ${ }^{60}$ and later Mattor and Diamond ${ }^{61}$ in the MFE context) becomes advantageous.

Up until now, we have not addressed the origin of symmetry breaking, i.e., the key question of what sets the net mean $k_{\|}$(i.e., $\left\langle k_{\|}\right\rangle$), which appears so prominently in the wave-momentum flux. Indeed, $\left\langle k_{\|}\right\rangle$also plays an important role in the resonant particle momentum flux, particularly in the residual stress. One advantage of the general framework we present here is that it allows us to address and compare several possible symmetry breaking mechanisms and quantitatively explore the competition between them. In contrast, all previous works have postulated a single symmetry breaking mechanism a priori.

The approach to $\left\langle k_{\|}\right\rangle$is best formulated by considering the mean field wave kinetic equation for $\langle N\rangle$, which is

$$
\begin{aligned}
\frac{\partial\langle N\rangle}{\partial t} & +\frac{1}{r} \frac{\partial}{\partial r}\left[r\left\langle\left(v_{\mathrm{gr}}+V_{r}\right) N\right\rangle\right]-\frac{\partial}{\partial k_{r}}\left[k_{\theta}\left\langle v_{E}\right\rangle^{\prime}\langle N\rangle\right] \\
& -\frac{\partial}{\partial k_{\|}}\left(k_{\|}\left\langle v_{\|}\right\rangle\langle\langle N\rangle)\right. \\
& =\nabla_{\mathbf{k}} \cdot\left[\overleftrightarrow{\mathbf{D}_{k}} \cdot \nabla_{\mathbf{k}}\langle N\rangle\right]+2 \gamma_{\mathbf{k}}\langle N\rangle+\left\langle C_{\mathrm{NL}}(N)\right\rangle,
\end{aligned}
$$

where the first term on the RHS accounts for random straining, the second for linear wave growth and the third for nonlinear wave-wave scattering. Then, taking $\int d \mathbf{k} k_{\|}$to obtain a general evolution equation for $\left\langle k_{\|}\right\rangle$yields

$$
\begin{aligned}
\frac{\partial}{\partial t}\left\langle k_{\|}\right\rangle= & -\frac{1}{r} \frac{\partial}{\partial r}\left[r\left\langle\Pi_{r, \|}^{w}\right\rangle\right]-\int d \mathbf{k}\left(\frac{\partial k_{\|}}{\partial k_{r}}\right) k_{\theta}\left\langle v_{E}\right\rangle^{\prime}\langle N\rangle \\
& -\int \mathbf{k} \hat{\mathbf{b}} \cdot \overleftrightarrow{\mathbf{D}_{\mathbf{k}}} \cdot \nabla_{\mathbf{k}}\langle N\rangle+2 \int d \mathbf{k} k_{\|} \gamma_{\mathbf{k}}\langle N\rangle-\gamma_{\mathrm{NL}}\left\langle k_{\|}\right\rangle .
\end{aligned}
$$

Here the last term on the RHS reflects the net decay of wave momentum by coupling to dissipation via nonlinear interaction of all sorts. Heuristically $\gamma_{\mathrm{NL}}$ should be thought of as a nonlinear decay rate for parallel wave momentum. Clearly $\gamma_{\mathrm{NL}}$ is comparable to $\nu_{T}$ but not necessarily precisely equal to it. Thus, at steady state, $\left\langle k_{\|}\right\rangle$satisfies

$$
\begin{aligned}
\gamma_{\mathrm{NL}}\left\langle k_{\|}\right\rangle= & -\frac{1}{r} \frac{\partial}{\partial r}\left[r\left\langle\Pi_{r, \|}^{w}\right\rangle\right]-\int d \mathbf{k}\left(\frac{\partial k_{\|}}{\partial k_{r}}\right) k_{\theta}\left\langle v_{E}\right\rangle^{\prime}\langle N\rangle \\
& -\int d \mathbf{k} \hat{\mathbf{b}} \cdot \overleftrightarrow{\mathbf{D}_{\mathbf{k}}} \cdot \nabla_{\mathbf{k}}\langle N\rangle+2 \int d \mathbf{k} k_{\|} \gamma_{\mathbf{k}}\langle N\rangle
\end{aligned}
$$

so the net local wave momentum density is determined by the competition between nonlinear decay and: 


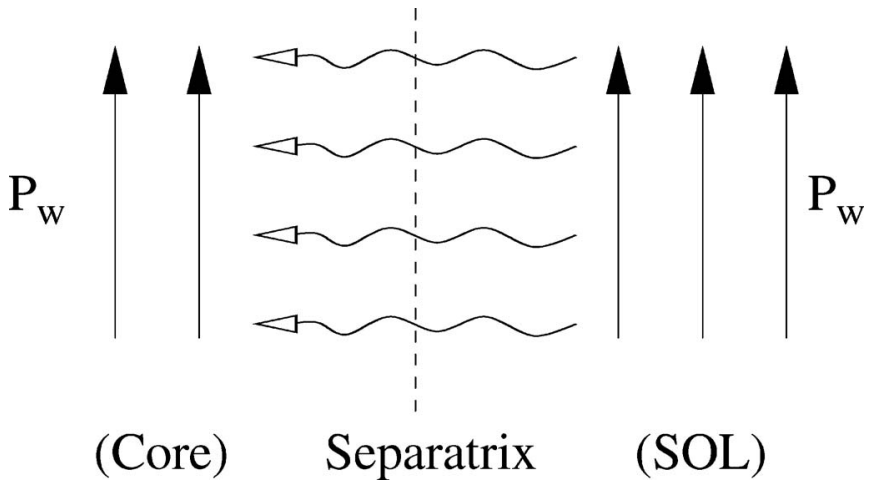

FIG. 4. Sketch of influx of wave momentum from the SOL.

(i) The local inflow or outflow of wave momentum density by transport, given by $-\nabla_{r} \cdot\left\langle\Pi_{r, \|}^{w}\right\rangle$. For example this process would account for growth of local wave momentum density in the core plasma as a result of influx from an edge or SOL momentum source, as depicted in Fig. 4.

(ii) The enhancement of $\left\langle k_{\|}\right\rangle$via the synergistic interaction of $\mathbf{E} \times \mathbf{B}$ shearing $\left(\left\langle v_{E}\right\rangle^{\prime}\right)$ and magnetic shear (required for $\left.\partial k_{\|} / \partial k_{r} \neq 0\right)$. This effect, represented by $-\int d \mathbf{k}\left(\partial k_{\|} / \partial k_{r}\right) k_{\theta}\left\langle v_{E}\right\rangle^{\prime}\langle N\rangle$, captures the now wellknown process of $E \times B$ shear-generated shifting of the spectrum off the resonant surface, which has been studied by many authors. ${ }^{27,62}$ In the eikonal theory formulation given here, that process appears naturally as one whereby $k_{\|}$"winds up" in response to an increase in $k_{r}$ induced by mean velocity shear. Magnetic shear (i.e., $\partial k_{\|} / \partial k_{r} \neq 0$ ) is absolutely essential to the viability of this process. Note also that since $\partial k_{\|} / \partial k_{r}$ $\sim k_{\theta}$, the overall term $\sim k_{\theta}^{2}$, so no additional poloidal symmetry breaking is required.

(iii) The input of wave momentum by the stochastic refractive force. This mechanism is driven by fluctuating mesoscale flows with parallel structure induced by poloidal asymmetry. Geodesic acoustic modes (GAMs) are thus a natural candidate. Hence, symmetry breaking by the GAM-induced refractive force is a possible wave momentum source in the plasma edge, where GAM activity is strong. The relation between GAM activity and intrinsic rotation has not been explored and merits further study.

(iv) Asymmetry in growth. This effect captures the possibility that one sign of $k_{\|}$may be preferred, dynamically. Such a phenomenon can occur when parallel velocity shear is present. This mechanism has been advocated by Coppi. ${ }^{63}$

The symmetry-breaking mechanisms are summarized in Table II. A virtue of this general approach to the question of symmetry breaking is that it allows quantitative comparisons between competing mechanisms. For example, by comparing the first and second terms on the RHS of Eq. (36b), one could determine the strength of the electric field shear required for the "shearing" mechanism of local symmetry breaking to exceed the influx of net wave momentum from
TABLE II. Mechanisms of $k_{\|}$-symmetry breaking.

(i) Radial flux of wave momentum

- possible influx of momentum from scrape off layer

- potentially critical in edge region, close to symmetry

breaking

(ii) $\mathbf{E} \times \mathbf{B}$ shearing

- requires magnetic shear, i.e., $\partial k_{\|} / \partial k_{r} \neq 0$

- critical in or near barrier regions, either pedestal or

ITB, but not limited to these

(iii) Growth asymmetry $\left[\gamma\left(+k_{\|}\right) \neq \gamma\left(-k_{\|}\right)\right]$

- may enter via parallel velocity shear

(iv) Refraction by GAMs

- likely significant near edge

- largely unexplored

neighboring regions, such as the edge. Comparisons such as these will be very useful in understanding the dynamics of momentum transport bifurcation, changes in intrinsic rotation and the relation of these two phenomena to changes in confinement.

This section has presented a rather lengthy reformulation of the quasilinear theory of momentum transport by nonresonant particles in terms of wave-momentum transport. The skeptical (and tired!) reader is no doubt wondering "just what have we gained by all this?." The answer to this very reasonable question is threefold, namely:

(i) Since nonresonant transport is fundamentally a wave process (i.e., associated with particle "sloshing"), it should, in principle, be formulated in terms of wave dynamics. This approach, in the vein of radiative transfer and radiation hydrodynamics, accomplishes precisely that! Ultimately, nonresonant transport is related to wave population gradients in both $\mathbf{x}$ and $\mathbf{k}$, with radiative transport coefficients and correlation times set by wave-wave interaction. A novel offdiagonal momentum flux contribution driven by $\left\langle v_{E}\right\rangle^{\prime}$ was identified, as well.

(ii) The origin of the irreversibility which underpins the stationary state counterpart of the nonresonant quasilinear flux is clearly identified.

(iii) A systematic way to assess the relative strength and importance of several possible competing symmetry breaking mechanisms has been developed.

Finally we note that these ideas, approaches, and methods may also be useful in the study of nonlocality phenomena in turbulent transport. In particular, they suggest one route toward a systematic theory of turbulence spreading.

\section{CALCULATING THE RESONANT PARTICLE MOMENTUM FLUX}

In this section, we present the calculation of the parallel momentum flux carried by resonant particles. In contrast to the calculation of the nonresonant portion, calculating the resonant particle momentum flux is relatively straightforward and follows from a direct application of methods familiar from quasilinear theory. 
The quasilinear gyrokinetic equation for the evolution of the resonant particle (i.e., ion) momentum is just

$$
\frac{\partial}{\partial t}\left\langle P_{\|}\right\rangle_{R}+\frac{1}{r} \frac{\partial}{\partial r}\left[r\left\langle\Pi_{r, \|}^{R}\right\rangle\right]=\int d^{3} v \dot{v}_{\|} \widetilde{f}_{i}^{R},
$$

where the resonant particle momentum flux is

$$
\Pi_{r, \|}^{R}=\left\langle\widetilde{v}_{E r} \widetilde{P}_{\| i}\right\rangle=m_{i} n_{0} \int d^{3} v v_{\|} \frac{d r}{d t} \widetilde{f}_{i}^{R}
$$

and $\widetilde{f}_{i}^{R}$ is the resonant, linear ion response. As shown in Sec. II, the RHS corresponds to momentum exchange between waves and particles and cancels identically with its counterpart involving $C_{w-p}(N)$ in the $k_{\|}$moment of the wave kinetic equation. Hence, the RHS is hereafter neglected, and we need only focus on $\left\langle\Pi_{r, \|}^{R}\right\rangle$. As usual in quasilinear theory, we simply plug the resonant linear response $\widetilde{f}_{i}^{R}$ into $\left\langle\Pi_{r, \|}^{R}\right\rangle$ to calculate the flux. The linear response $\widetilde{f}_{i, \mathbf{k}}^{R}$ is

$$
\tilde{f}_{i, \mathbf{k}}^{R}=\frac{-J_{0}(\lambda)}{\omega_{k}-\omega_{E B}-k_{\|} v_{\|}+i \epsilon} c_{s}^{2}\left\{\frac{k_{\theta}}{\omega_{c i}} \frac{\partial\left\langle F_{i}\right\rangle}{\partial x}+k_{\|} \frac{\partial\left\langle F_{i}\right\rangle}{\partial v_{\|}}\right\} \frac{e \widetilde{\phi}_{\mathbf{k}}}{T_{e}},
$$

where $\lambda \equiv k_{\perp} \rho_{\perp}$ and $\omega_{E B} \equiv k_{\theta}\left\langle v_{E}\right\rangle$. After considerable (albeit straightforward) algebra we obtain the resonant ion momentum flux [whose form agrees with Eqs. (26a) and (26b) above]

$$
\left\langle\Pi_{r, \|}^{R}\right\rangle=n_{0} m_{i}\left[-\chi_{\phi} \frac{\partial}{\partial r}\left\langle v_{\|}\right\rangle+V_{r}\left\langle v_{\|}\right\rangle+S\right],
$$

where for a shifted Maxwellian $\langle f\rangle$,

$$
\begin{aligned}
\chi_{\phi}= & \sqrt{2 \pi} \sum_{\mathbf{k}}\left(k_{\theta} \rho_{i}\right)^{2} \frac{v_{t i}}{\left|k_{\|}\right|} \Omega^{2} \Gamma_{0}(b) e^{-\Omega^{2}}\left|\frac{e \tilde{\Phi}_{\mathbf{k}}}{T_{i}}\right|^{2}, \\
V_{r}= & \sqrt{\frac{\pi}{2}} \sum_{\mathbf{k}}\left(k_{\theta} \rho_{i}\right)^{2} \frac{v_{t i}}{\left|k_{\|}\right|} \frac{1}{L_{n}} \Gamma_{0}(b)\left|\frac{e \widetilde{\Phi}_{\mathbf{k}}}{T_{i}}\right|^{2} e^{-\Omega^{2}} \\
& \times\left\{1-\frac{\eta_{i}}{\eta_{i}^{\text {crit }}}-\frac{\bar{\omega}_{\mathbf{k}}}{\omega *_{i}}-\eta_{i} \Omega^{2}\right\}
\end{aligned}
$$

and

$$
\begin{aligned}
S= & \sqrt{\pi} \sum_{\mathbf{k}}\left(k_{\theta} \rho_{i}\right)^{2} \frac{v_{t i}^{2}}{L_{n}} \frac{1}{\left|k_{\|}\right|} \Omega \Gamma_{0}(b)\left|\frac{e \widetilde{\Phi}_{\mathbf{k}}}{T_{i}}\right|^{2} e^{-\Omega^{2}} \\
& \times\left\{1-\frac{\eta_{i}}{\eta_{i}^{\text {crit }}}-\frac{\bar{\omega}_{\mathbf{k}}}{\omega *_{i}}-\eta_{i} \Omega^{2}\right\} .
\end{aligned}
$$

Here the notation is standard, so $\eta_{i}^{\text {crit }}=2\left[1+2 b\left(1-I_{1} / I_{0}\right)\right]^{-1}$, $\Omega=\bar{\omega}_{\mathbf{k}} / \sqrt{2} k_{\|} v_{t i}, b=k_{\perp}^{2} \rho_{i}^{2}, I_{0}$ and $I_{1}$ are the modified Bessel functions, $v *_{e, i}$ are the electron and ion diamagnetic velocities, $\bar{\omega}_{k}$ is the Doppler shifted wave frequency defined by $\omega_{\mathbf{k}}-\omega_{E B} \equiv \bar{\omega}_{\mathbf{k}}+k_{\|}\left\langle v_{\|}\right\rangle$, and $\omega *_{e, i}=k_{\theta} v *_{e, i}, \quad \tau=T_{e} / T_{i}, \quad \Gamma_{0}$ $=I_{0} e^{-b}$, etc.

Some discussion of the transport coefficients $\chi_{\phi}, V_{r}$, and $S$ is appropriate at this point. Predictably, $\chi_{\phi} \sim \chi_{i}$ but $\chi_{\phi} \neq \chi_{i}$, on account of the structure of resonant coupling to the spectrum of wave phase velocities. $\chi_{\phi}$ (and obviously, the entire resonant particle flux) decays rapidly $\left[\sim \exp \left(-\Omega^{2}\right)\right]$ for nonresonant particles, and may be written as $\chi_{\phi} \sim\left\langle\widetilde{v}_{E}^{2}\right\rangle \tau_{a c}$, where $\tau_{a c}$ is set by the dispersion in the distribution of the ion transit rate. Of course, $\chi_{\phi} \sim D_{\mathrm{GB}}$ for "mixing length estimate" fluctuation levels. The convection velocity $V_{r}$ is rather sensitive and model dependent. $V_{r}$ is inward (corresponding to a pinch) for ITG modes in the resonant regime near threshold $(|\Omega| \lesssim 1)$. For regimes far from threshold $(\Omega \gg 1) V_{r}$ is necessarily outward, however as noted above, the magnitude of this term is then negligible. Near marginality, but for electron drift waves, the sign of the convective term depends sensitively on $\eta_{i}$, and requires a quantitative description of the microturbulence spectrum. Note that the convective term scales as $V_{r} / \chi_{\phi} \sim 1 /\left(\Omega^{2} L_{\perp}\right)$, a profile scale length. Thus, the resonant particle pinch complements the nonresonant turbulent convection (TurCo) pinch, ${ }^{23}$ derived for toroidal geometry. The latter is nonresonant (and so must represent wave transport), is inward for electron drift waves, can attain either sign (depending on plasma parameters) for ITG modes and has $V_{r} / \chi_{\phi} \sim 1 / R$ [i.e., $O(\epsilon)$ smaller], but is insensitive to the resonance function. In other words, if the percentage weighting of resonant particles is lower than $\epsilon$, TurCo provides the main convection effect. On the other hand, near marginal stability, where the percentage of resonant particles is high, the resonant particle pinch is the primary cause of convection. Furthermore, near marginality $\left|\bar{\omega}_{\mathbf{k}} \ll \omega *_{i}\right|$ and $\left|\Omega^{2}\right| \lesssim 1$, so that

$$
\frac{V_{r}}{\chi_{\phi}} \approx \frac{1}{\Omega^{2}} \frac{1}{L_{n}}\left\{1-\frac{\eta_{i}}{\eta_{i}^{\text {crit }}}\right\},
$$

so that for unstable ITG, the $\nabla T_{i}$ driven pinch is inward in rough agreement with Ref. 21 , but the $\nabla n$ driven pinch is outward, opposite to the nonresonant pinch predicted in Ref. 22. Considering now the third term in the resonant momentum flux given by Eq. (39a), since $S / V_{r} \approx \Omega v_{t i}, S$ must vanish in the absence of symmetry breaking (i.e., $S \rightarrow 0$ as $\left.\left\langle k_{\|}\right\rangle \rightarrow 0\right)$. It is also clear, for regimes of finite symmetry breaking, the sign of the residual stress follows identical rules to that of the resonant particle convection term, but multiplied by $\operatorname{sgn}\left(\left\langle k_{\|}\right\rangle\right)$, which is determined by Eq. (36a). Also, it is readily apparent that the residual stress is a much broader concept than that encountered while considering $\mathbf{E} \times \mathbf{B}$ shearing effects on momentum transport. More generally, it is clear that $S$ emerges naturally while constructing the quasilinear theory of momentum transport in the presence of symmetry breaking $\left(\left\langle k_{\|}\right\rangle \neq 0\right)$.

Here we note that the above result is in agreement with a similar calculation of the resonant particle flux performed in Ref. 21. However, we emphasize that while the resonant particle flux contains a host of tantalizing nondiffusive terms, neglecting the wave momentum flux - as was done in Ref. 21 - is in general not justified since the relative magnitude of the resonant particle flux in comparison to the nondiffusive wave momentum flux is strongly parameter dependent. Note that the results in Ref. 21 do not exhibit an apparent modedependency which is obvious from the standard calculations using the gyrokinetic equation. We also note that the basic scaling $V_{r} / \chi_{\phi} \sim 1 / \Omega^{2}\left(\Omega^{2} L_{\perp}\right)$ is suggestive of an underlying 
physical mechanism which does not depend on toroidicity (i.e., exists in simpler geometry), leaving some doubts on the scaling with respect to $L_{n}$ proposed in Ref. 22. Furthermore, the critical role of wave momentum is highlighted when one considers that by neglecting this component, the RHS of Eq. (37a) (which corresponds to wave-particle interactions, and is generally nonzero) is not cancelled by its counterpart in the wave momentum equation. Thus, any description of momentum transport purely in terms of resonant particle evolution leaves an unaccounted for sink/source of momentum in the mean momentum evolution equation! Finally, it should be noted that this analysis has not addressed the nature of particle transport or the nonadiabatic electron response. Considering these will surely introduce additional momentum transport effects related to the interplay of particle and momentum transport. Such cross coupling is discussed further in Refs. 24 and 25.

\section{IMPLICATIONS FOR MOMENTUM TRANSPORT PHENOMENOLOGY}

Until now, we have focused on the general structure of the theory of momentum transport. Here we discuss the implications for and applications of, the theory to several aspects of the phenomenology of toroidal momentum transport in tokamaks. We proceed by first summarizing the structure of the predicted momentum flux, first discussing its implication for quasistationary momentum transport phenomena and then for momentum transport bifurcation phenomena. Detailed modeling of specific cases is left to future papers. Here, rather, we explore the general scope of potential applications which are possible within this theory.

The principal results obtained so far are the stationary state values of the radial flux of parallel momentum $\left\langle\Pi_{r, \|}^{w}\right\rangle$, the evolution equation for $\left\langle k_{\|}\right\rangle$(which links symmetry breaking to dynamics), and the resonant particle parallel momentum flux $\left\langle\widetilde{v}_{E r} \widetilde{P}_{\|}\right\rangle_{R}$. These are, respectively,

$$
\left\langle\Pi_{r, \|}^{w}\right\rangle=\int d \mathbf{k} k_{\|}\left\{\left\langle V_{r}\right\rangle N-\frac{v_{\mathrm{gr}}^{2}}{\nu_{T_{\mathbf{k}}}} \frac{\partial\langle N\rangle}{\partial r}+v_{\mathrm{gr}} \frac{k_{\theta}}{\nu_{T_{\mathbf{k}}}}\left\langle v_{E}\right\rangle^{\prime} \frac{\partial\langle N\rangle}{\partial k_{r}}\right\},
$$

$$
\begin{aligned}
& \frac{\partial}{\partial t}\left\langle k_{\|}\right\rangle+\gamma_{\mathrm{NL}}\left\langle k_{\|}\right\rangle=-\frac{1}{r} \frac{\partial}{\partial r}\left[r\left\langle\Pi_{r, \|}^{w}\right\rangle\right] \\
&-\int d \mathbf{k}\left(\frac{\partial k_{\|}}{\partial k_{r}}\right) k_{\theta}\left\langle v_{E}\right\rangle^{\prime}\langle N\rangle \\
&-\int d \mathbf{k} \hat{\mathbf{b}} \cdot \overleftrightarrow{\mathbf{D}_{\mathbf{k}}} \cdot \nabla_{\mathbf{k}}\langle N\rangle, \\
&\left\langle\widetilde{v}_{E r} \widetilde{P}_{\|}\right\rangle_{R}=n_{0} m_{i}\left[-\chi_{\phi}^{R} \frac{\partial}{\partial r}\left\langle v_{\|}\right\rangle+V_{r}^{R}\left\langle v_{\|}\right\rangle+S_{R}\right],
\end{aligned}
$$

where

$$
\chi_{\phi}^{R}=\sqrt{2 \pi} \sum_{k}\left(k_{\theta} \rho_{i}\right)^{2} \frac{v_{t i}}{\left|k_{\|}\right|} \Omega^{2} \Gamma_{0}(b) e^{-\Omega^{2}}\left|\frac{e \Phi_{\mathbf{k}}}{T_{i}}\right|^{2},
$$

$$
\begin{aligned}
V_{r}= & \sqrt{\frac{\pi}{2}} \sum_{\mathbf{k}}\left(k_{\theta} \rho_{i}\right)^{2} \frac{v_{t i}}{\left|k_{\|}\right|} \frac{1}{L_{n}} \Gamma_{0}(b)\left|\frac{e \widetilde{\Phi}_{\mathbf{k}}}{T_{i}}\right|^{2} e^{-\Omega^{2}} \\
& \times\left\{1-\frac{\eta_{i}}{\eta_{i}^{\text {crit }}}-\frac{\bar{\omega}_{\mathbf{k}}}{\omega *_{i}}-\eta_{i} \Omega^{2}\right\}, \\
S= & \sqrt{\pi} \sum_{\mathbf{k}}\left(k_{\theta} \rho_{i}\right)^{2} \frac{v_{t i}^{2}}{L_{n}} \frac{1}{\left|k_{\|}\right|} \Omega \Gamma_{0}(b)\left|\frac{e \widetilde{\Phi}_{\mathbf{k}}}{T_{i}}\right|^{2} e^{-\Omega^{2}} \\
& \times\left\{1-\frac{\eta_{i}}{\eta_{i}^{\text {crit }}}-\frac{\bar{\omega}_{\mathbf{k}}}{\omega *_{i}}-\eta_{i} \Omega^{2}\right\} .
\end{aligned}
$$

The total turbulent momentum flux is conventionally expressed for purposes of data analysis and modeling in the form

$$
\left\langle\Pi_{r, \|}^{T}\right\rangle=n_{0} m\left[-\chi_{\phi} \frac{\partial}{\partial r}\left\langle v_{\phi}\right\rangle+V_{r}\left\langle v_{\phi}\right\rangle+S\right],
$$

where $\chi_{\phi}$ is the diffusivity (i.e., turbulent viscosity accounting for the radial scattering of toroidal flow), $V_{r}$ is the radial convection velocity (i.e., pinch, if inward), and $S$ is the residual stress, which arises since waves and particles can exchange momentum. $S$ has no counterpart in the particle flux. Loosely speaking, anomalous electron-ion energy coupling might be thought of as a process analogous to $S$ in the energy balance, though residual stress accounts for ion-wave momentum exchange, rather than ion-electron momentum exchange. Both anomalous thermal transfer and residual stress (related to anomalous momentum transfer) are processes mediated by waves. Pending possible re-expression of $\left\langle\Pi_{r, \|}^{w}\right\rangle$ in terms of $\left\langle v_{\|}\right\rangle$, Eq. (40a) implies that

$$
\begin{aligned}
& \chi_{\phi}=\chi_{\phi}^{R}, \\
& V_{r}=V_{r}^{R}, \\
& S=S_{R}+\left\langle\Pi_{r, \|}^{w}\right\rangle
\end{aligned}
$$

so the momentum diffusivity and the convection velocity are carried primarily by resonant particles, while the residual stress is supported by both resonant particles and waves. Note that the residual stress is usually neglected in traditional " $D$ and $V$ models," but can indeed be the dominant offdiagonal contribution to momentum transport, particularly in regimes of strong turbulence away from marginality. Surely the most basic message of this section is the need to address the residual stress contribution to the momentum flux.

We first discuss the implications of the theory for transport of momentum in quasistationary states. Obviously, $\chi_{\phi} \sim \chi_{i}$ but $\chi_{\phi} \neq \chi_{i}$, in accord with all experimental results. Inward convection (i.e., $V_{r}<0$, a momentum pinch) due to resonant transport is predicted for profiles which are "stiff" to ITG turbulence (i.e., near marginal). Here $V / \chi_{\phi} \sim 1 / L_{\perp}$, but we must emphasize that both the magnitude and the sign of $V_{r}$ are very sensitive to parameter variations and must be studied quantitatively in order to meaningfully apply the theory to perturbative transport experiments, such as those of Yoshida et al. on JT-60U. ${ }^{12}$ We again note that the resonant ion pinch discussed here is complementary to the nonreso- 
nant TurCo pinch discussed in Ref. 23. It is very important, however, to note that the residual stress $S$ can drive inward nondiffusive momentum transport in addition to $V_{r}$. Both resonant and nonresonant particles can contribute to $S$. A nearly universal contribution to $S$ is the radiative diffusive flux of wave momentum $\left(\sim-D_{\text {rad }} \partial\left\langle P_{\|}^{w}\right\rangle / \partial r\right)$, which is inward, since fluctuation intensity tends to peak toward the outside [i.e., $\langle N\rangle$ and $\left\langle P_{\|}\right\rangle$increase while $T(r)$ and $n(r)$, etc. decrease]. Also, the edge region is replete with potentially viable symmetry breaking mechanisms, so $\left\langle k_{\|} \neq 0\right\rangle$ there. Thus, inward diffusion of wave momentum is clearly a robust candidate mechanism to support residual stress contributions to the off-diagonal momentum flux. However, we hasten to add that electric field shear may also contribute to the inward momentum flux via $S$, as should be apparent from Eq. (40a). Indeed note that $\left\langle v_{E}\right\rangle^{\prime}$ can drive a nondiffusive momentum flux even if electric field shear is not the principal symmetry-breaking mechanism which determines $\left\langle k_{\|}\right\rangle$. This is due to the fact that flux and symmetry breaking of $\left\langle k_{\|}\right\rangle$are distinct in this theory, though both operate via $\left\langle v_{E}\right\rangle^{\prime}$.

Turning to the interesting phenomenon of momentum transport bifurcations, we focus primarily on the wave momentum flux, since this is the most relevant to strong turbulence regimes from which bifurcations initiate, and since $\left\langle\Pi_{r, \|}^{w}\right\rangle$ is clearly sensitive to electric field shear, which is a key bifurcation order parameter. We remind the reader that momentum transport bifurcations related to a change in the structure of the momentum flux have been observed both at the edge and in the core of tokamak plasmas. In general, it is clear from Eqs. (40a) and (40b) that both electric field shear and fluctuation profile affects are important in regulating the local wave momentum flux. Electric field shear enters both as a symmetry breaking mechanism, which along with magnetic shear renders $\left\langle k_{\|}\right\rangle \neq 0 .\left\langle v_{E}\right\rangle^{\prime}$ also acts as a direct driver of $\left\langle\Pi_{r, \|}^{w}\right\rangle$ with the multiplicative factor $k_{\theta} \partial v_{\mathrm{gr}} / \partial k_{r}$, which is mode phase velocity dependent. Of course, electric field shear also regulates fluctuation levels. Thus, we see that a generic scaling is $\left\langle\Pi_{r,,}^{w}\right\rangle \sim \hat{s}\left(\left\langle v_{E}\right\rangle^{\prime}\right)^{\alpha} v_{\mathrm{ph}}\langle I\rangle$, where $\alpha \sim 1 \rightarrow 2$. Here $I$ is the fluctuation intensity level, $I(r)$. The fluctuation profile structure, often associated with the edge, is also very relevant. Radiative diffusion of wave momentum $\left(\sim-D_{\text {rad }} \partial\left\langle P_{\|}\right\rangle / \partial r\right)$ can produce an influx of wave momentum from the edge into the core. Similarly, $-\nabla \cdot\left\langle\Pi_{r, \|}^{w}\right\rangle$ can also act to produce or enhance symmetry breaking (i.e., $\left.\left\langle k_{\|}\right\rangle\right)$and wave momentum transport via an inward wave momentum flux. Since these intensity gradient driven fluxes compete with the electric field shear driven convective flux of wave momentum to regulate the overall structure of $\left\langle\Pi_{r, \|}^{w}\right\rangle$, any changes in the balance between the competitors may trigger possible momentum transport bifurcations.

Given the rich structure of $\left\langle\Pi_{r, \|}^{w}\right\rangle$, one can identify at least two scenarios for momentum transport bifurcations. These are:

(i) A change in $\left\langle\Pi_{r, \|}^{w}\right\rangle$ from an intensity diffusion dominated state $\left(\Pi_{r, \|}^{w} \sim-D_{\text {rad }} \partial\left\langle P_{\|}\right\rangle / \partial r\right)$ to a $\left\langle v_{E}\right\rangle^{\prime}$-driven convection dominated state $\left[\Pi_{r, \|}^{w} \sim\left(k_{\theta} \partial v_{\mathrm{gr}} / \partial k_{r}\right)\right.$ $\left.\times\left\langle v_{E}\right\rangle^{\prime}\left\langle P_{\|}\right\rangle / \nu_{T}\right]$. (ii) A change in a convection dominated state of $\left\langle\Pi_{r, \|}^{w}\right\rangle$ due to a change in the product $v_{\mathrm{ph}}\left\langle v_{E}\right\rangle^{\prime}$. This could occur either via an electric field bifurcation or via a change in $v_{\mathrm{ph}}$, the turbulence phase velocity.

Type (i) qualitatively resembles an $L \rightarrow H$ transition, which occurs at the edge and is associated with an increase in electric field shear so that $V_{w}\langle N\rangle$ increases while $-D_{\text {rad }} \partial\langle N\rangle / \partial r$ drops. In this scenario, $\left\langle k_{\|}\right\rangle$is set by $\left\langle v_{E}\right\rangle^{\prime}$, as well, strengthening the dependence on $\left\langle v_{E}\right\rangle^{\prime}$. Type (i) bifurcations seem consistent with the observed strong correlation between scaling trends in intrinsic rotation and the $L \rightarrow H$ transition. Type (ii) bifurcations qualitatively resemble internal momentum transport bifurcations, such as those that occur with ITBs and as that observed recently on the TCV tokamak. ${ }^{16}$ Such internal bifurcations can occur via an increase in local $\left\langle v_{E}\right\rangle^{\prime}$ in the core, thus increasing both convective wave momentum flux and symmetry breaking. This scenario is relevant to ITBformation, where changes in the momentum flux would be accompanied by improvements in confinement. However, the theory suggests that internal momentum transport bifurcations can also occur via changes in $v_{\mathrm{ph}}$, thereby reversing the direction of wave momentum convection. This scenario is especially relevant to the core plasma, where intensity gradients are weak, and to instances where the change in momentum transport is not accompanied by a noticeable change in confinement. This is the case in a recently reported, interesting experiment on TCV, during which core rotation "flipped" sign from counter to co-current direction, as the density was increased in Ohmic plasmas (N.B. similar phenomena have been observed on Alcator C-Mod ${ }^{64}$ ). No significant change in thermal transport was observed. We speculate that the observed momentum transport bifurcation could be explained as a change in $v_{\mathrm{ph}}$ from $v *_{e}$ direction to $v *_{i}$ direction, while the ambient fluctuation population changes from predominantly electron drift waves to predominantly ITG modes, as density increases while approaching the $\mathrm{OH}$-saturation regime. Indeed, excitation of ITG turbulence has long been thought to explain the saturation of energy confinement in high density Ohmic plasmas. Detailed, quantitative modelling of this fascinating momentum transport phenomenon using the theory presented here is ongoing, and will be discussed in a future publication. One interesting question which is stimulated by the TCV results is that while the experiment clearly exhibits hysteresis phenomena in density, in that co-direction rotation persists when density is lowered below $\mathrm{OH}$-saturation levels, the theory suggests a smooth evolution with $v *$ as it changes from $v *_{i}$ to $v *_{e}$. Put another way, the theory seems to predict a second order transition while the data suggests a first order transition. We speculate that a possible resolution of this dilemma is "turbulence spreading" or turbulent overshoot of the ITG population into the electron drift wave population. Turbulence intensity fronts are well known to exhibit "effective inertia,"65 thus enabling them to continue to advance when local drive is lowered. We suggest that the dynamics may be described by two coupled nonlinear diffusion equations for the electron and ion drift wave quanta density. In the past, such simple nonlinear diffusion equations have had some success in mod- 
elling the nonlocal dynamics of turbulence spreading. Constructing a credible theoretical model of the two interacting turbulence populations and then using that model to explain the observed density hysteresis constitute very significant challenges to our ongoing studies of TCV phenomenology. Finally, we comment that a theoretical understanding of the implications of reversed or weakly negative magnetic shear for the $\left\langle v_{E}\right\rangle^{\prime}$-driven convective wave momentum flux would be of great interest in elucidating intrinsic rotation in ITB plasmas. This will be pursued in future works.

\section{CONCLUSIONS}

In this paper, we have presented a general theory of parallel momentum transport by collisionless electrostatic drift wave turbulence. The principal results of this paper are:

(i) The proof of a general momentum conservation theorem, which relates total mean momentum evolution to the resonant particle momentum flux, the wave momentum flux, and the refractive force exerted by flow structures, has been derived. This theorem accounts for, and resolves all ambiguities related to the interaction between momentum transport by turbulence and momentum transfer by wave-particle interaction. The physics of the fluctuation-dependent momentum source/sink which appears in the naive quasilinear theory is clarified.

(ii) The calculation of the wave momentum flux valid for stationary turbulence. The theoretical formulation which we present yields a general expression for the radial flux of parallel wave momentum, as given by Eq. (40a). The wave momentum flux contains both radiative diffusive flux and a novel $\left\langle v_{E}\right\rangle^{\prime}$-driven convective flux. The wave momentum flux contributes to the total residual stress, i.e., that part of the momentum flux not explicitly proportional to $\left\langle v_{\phi}\right\rangle$ or $\partial\left\langle v_{\phi}\right\rangle / \partial r$. All the usual ambiguities associated with calculating nonresonant parallel momentum transport have been resolved.

(iii) The calculation of the resonant particle momentum flux, as given by Eqs. (40c)-(40f). The resonant momentum flux consists of a diffusive flux (with $\chi_{\phi} \sim \chi_{i}$ but $\chi_{\phi} \neq \chi_{i}$ ), a novel convective flux which may be inward in certain regimes, and a novel residual stress piece, driven by $\nabla T_{i}$ and explicitly dependent upon the symmetry breaking mechanism [i.e., the origin of $\left\langle k_{\|}\right\rangle \neq 0$, see (v) below].

(iv) The calculation of the refractive force, which is shown to depend sensitively on the structure of secondary parallel flows, such as those produced by GAMs, and on radial electric field shear.

(v) A general expression for the evolution of $\left\langle k_{\|}\right\rangle$, which allows evaluation and comparison of competing symmetry breaking mechanisms, as shown in Eq. (40b).

(vi) The application of the theory to outline two possible scenarios for momentum transport bifurcations. Both of these involve a change in the momentum flux balance, and arise from evolution of the convective com- ponent of the wave momentum flux, proportional to $v_{\mathrm{ph}}\left\langle v_{E}\right\rangle^{\prime}$. In one case, $\left\langle v_{E}\right\rangle^{\prime}$ rises, as in a conventional electric field shear bifurcation, leading to overall improvement in confinement. In the other, $v_{\mathrm{ph}}$ changes direction, due to a change in the propagation direction of the underlying drift waves, but $\left\langle v_{E}\right\rangle^{\prime}$ and confinement do not change. The second scenario may be relevant to the phenomena recently observed in the core of the TCV tokamak.

The theory presented here has several significant limitations. First, the model is purely electrostatic, so field momentum transport is not addressed. In the case of Alfvénic turbulence, such as that driven by Alfvén eigenmodes, the field momentum density can be expected to be comparable to the nonresonant particle momentum density, thus complicating the wave momentum budget, and possibly introducing an Abraham force on the plasma. ${ }^{66}$ Second, we have not addressed the edge boundary condition on the rotation profile, beyond entertaining the possibility of a possible influx of wave momentum from the SOL. This boundary condition is quite likely nontrivial, as SOL flows are significant, they can exert a stress on the core plasma, and can modify wave propagation and wave momentum in the SOL plasma. ${ }^{15}$ Third, the Krook model employed in Sec. III needs improvement. This leads one to confront the interesting theoretical question of how nonlinear interaction and transfer processes driven by cascading or modulational instability respond to perturbations, such as those induced by population gradients. An improved model of "turbulent collisions" is required, and an approach using a modified Leith model holds promise. ${ }^{67,68}$ Finally, the results in this paper indicate that the calculation of wave momentum transport cannot really be separated from the calculation of turbulence spreading and other nonlocality phenomena which influence turbulence envelope evolution. ${ }^{69,70}$

While this paper is theoretical, it does offer several ideas and results of potential interest to experimentalists. First it presents a complete calculation and discussion of the structure and physics of the momentum flux (i.e., resonant particle + wave) and identifies (quantitatively) the residual stress as a key contributor to momentum transport. The residual stress is ignored in traditional " $\mathrm{D}$ and $\mathrm{V}$ models," and requires dual perturbation experiments to elucidate it. Second, we present an improved calculation of a resonant particle momentum pinch, which may be relevant to collisionless core plasmas near ITG marginality. This pinch has $V_{r} / \chi_{\phi} \sim 1 / L_{T}$. Third, we identify two possible momentum transport bifurcation scenarios. Well resolved, localized fluctuation measurements could be used to test the momentum transport bifurcation scenario based on a change in fluctuation $v_{\mathrm{ph}}$. Experiments which address intrinsic rotation evolution in ITB/plasmas with steepen $\nabla P$ would be of interest, too. Fourth, we identify mean electric field shear and GAMs as likely agents for transmitting the refractive force, and suggest future studies of the relation between these quantities and intrinsic rotation. Fifth, we offer a framework within which to compare and contrast the strength and relevance of competing turbulence symmetry breaking mechanisms. 
Future work will focus on extensions of the theory and on applications to specific experiments.

\section{ACKNOWLEDGMENTS}

The authors would like to extend special thanks to Sergey Nazarenko for insightful explanation of the Leith model and its relevance to turbulent collisions and to Xavier Garbet, K. Itoh, and S.-I Itoh for discussions and collaboration on the subjects of momentum transport and nonlocality in turbulence. We also thank (in alphabetical order) C. Bourdelle, C. S. Chang, G. Falchetto, Ph. Ghendrih, F. Hinton, Z. Lin, M. Malkov, Y. Sarazin and the participants in the 2007 Festival du Theorie for stimulating discussions of the theory and (also in alphabetical order) K. Burrell, P. deVries, B. Duval, C. Hidalgo, Y. Kamada, S. Kaye, V. Parail, J. E. Rice, W. Solomon, and M. Yoshida for discussions of the experimental data.

This research was supported by DOE Grant No. FG0204ER 54738 (UCSD) and DOE Grant No. DE-AC02-76CHO-3073 (PPPL). We also acknowledge support from the Center for Gyrokinetic Particle Simulation of Turbulent Transport in Burning Plasma and the Center for Gyrokinetic Simulation of Energetic Particles. P.H.D. would also like to acknowledge support from the MEXT project on Structure Formation in Plasma and the hospitality of Kyushu University, where part of this work was performed.

\section{APPENDIX A: EXPLICIT DERIVATION OF WAVE ACTION DENSITY}

In this appendix we derive an explicit expression for the wave action density from the gyrokinetic equation, and relate it to momentum exchange between resonant particles and waves. The induced plasma response to an external electrostatic perturbation may be written as

$$
\begin{aligned}
\delta F_{k, \omega}^{\mathrm{ind}}= & \frac{J_{0}\left(k_{\perp} \rho_{\perp}\right)}{\omega-\omega_{E B}-k_{\|} v_{\|}+i \epsilon}\left\{\frac{c}{B}(\hat{\mathbf{b}} \times \mathbf{k}) \cdot \frac{\partial\left\langle F_{s}\right\rangle}{\partial \mathbf{x}}\right. \\
& \left.-\frac{q_{s}}{m_{s}} k_{\|} \frac{\partial\left\langle F_{s}\right\rangle}{\partial v_{\|}}\right\} \delta \phi_{k, \omega}^{\mathrm{tot}} \equiv L_{k, \omega} \delta \phi_{k, \omega}^{\mathrm{tot}},
\end{aligned}
$$

where $\delta \phi^{\text {tot }}=\delta \phi^{\text {ind }}+\delta \phi^{\text {ext }}$. Considering the gyrokinetic Poisson equation, an equation for the induced response may be written as

$$
\begin{aligned}
k_{\perp}^{2} \delta \phi_{k, \omega}^{\text {ind }}= & 4 \pi \sum_{s} q_{s} \int d^{3} \bar{v}\left\{J_{0}(\lambda) \delta F_{k, \omega}^{\text {ind }}\right. \\
& \left.+\left[J_{0}^{2}(\lambda)-1\right] \frac{q_{s} \delta \phi_{k, \omega}^{\text {ind }}}{T_{s}}\left\langle F_{s}\right\rangle\right\},
\end{aligned}
$$

where it is understood that $\delta F_{k, \omega}^{\text {ind }}$ is species dependent. Equation (A2) may be simplified via the following notation:

$$
\epsilon_{\perp}(k) k_{\perp}^{2} \delta \phi_{k, \omega}^{\text {ind }}=4 \pi \sum_{s} q_{s} \int d^{3} v^{\prime} J_{0}(\lambda) \delta F_{k, \omega}^{\text {ind }},
$$

where $\epsilon_{\perp}(k) \equiv 1+\sum_{s}\left(k_{D s}^{2} / k_{\perp}^{2}\right)\left[1-I_{0}\left(b_{s}\right) \exp \left(-b_{s}\right)\right]$. This permittivity may be profitably understood to correspond to a "gyrokinetic vacuum.,"71
After substitution of Eq. (A1) into Eq. (A3), the induced response of the electrostatic field can be written in terms of the external perturbations, yielding

$$
\begin{aligned}
(1+ & \left.\chi_{k, \omega}\right) \epsilon_{\perp}(k) k_{\perp}^{2} \delta \phi_{k, \omega}^{\text {ind }} \\
& =4 \pi \sum_{s} q_{s} \int d^{3} v^{\prime} J_{0}(\lambda) L_{k, \omega} \delta \phi_{k, \omega}^{\text {ext }} .
\end{aligned}
$$

Defining the susceptibility by

$$
\begin{aligned}
\chi_{k, \omega}= & -4 \pi \sum_{s} \frac{q_{s}}{\epsilon_{\perp}(k) k_{\perp}^{2}} \int d^{3} \bar{v} \frac{J_{0}^{2}(\lambda)}{\omega-\omega_{E B}-k_{\|} v_{\|}+i \epsilon} \\
& \times\left[\frac{c}{B}(\hat{\mathbf{b}} \times \mathbf{k}) \cdot \nabla\left\langle F_{s}\right\rangle-\frac{q_{s}}{m_{s}} k_{\|} \frac{\partial\left\langle F_{s}\right\rangle}{\partial v_{\|}}\right],
\end{aligned}
$$

and the dispersion relationship is defined as

$$
0=D_{k, \omega} \equiv 1+\chi_{k, \omega} .
$$

The growth rate may be approximated as

$$
\gamma_{k}=\left.\frac{-\operatorname{Im} D_{k, \omega}}{\partial D_{k, \omega} / \partial \omega}\right|_{\omega=\omega_{k}} .
$$

Finally, the rate at which momentum is exchanged between waves and resonant particles is given by the RHS of Eq. (37a), and may be rewritten in terms of the susceptibility as

$$
S^{R}=\frac{1}{4 \pi} \int d \mathbf{k} k_{\|} \operatorname{Im} \chi_{k, \omega_{k}} \epsilon_{\perp}(k) k_{\perp}^{2}\left|\delta \phi_{k}\right|^{2},
$$

utilizing Eqs. (A7) and (A6), this may be simplified, yielding

$$
S^{R}=-2 \int d \mathbf{k} k_{\|} \gamma_{k} N_{k},
$$

where $N_{k}$ is the wave action density, and is defined as

$$
\left.N_{k} \equiv \frac{1}{8 \pi} \frac{\partial D_{k, \omega}}{\partial \omega}\right|_{\omega=\omega_{k}} \epsilon_{\perp}(k) k_{\perp}^{2}\left|\delta \phi_{k}\right|^{2} .
$$

\section{APPENDIX B: PHYSICS OF THE REFRACTIVE FORCE}

This appendix presents a brief discussion of the basic physics of the refractive force. A pedagogical tactic employed here is to illustrate aspects of the refractive force in the context of concepts and results familiar from the theory of zonal flow generation.

Put most generally, the refractive force is simply the rate of change of wave momentum density induced by wave packet refraction. Since the evolution of wave momentum density is described by the $k$-moment of the wave kinetic equation, we have

$$
\frac{\partial}{\partial t} \mathbf{P}_{w}+\nabla \cdot \overleftrightarrow{\mathbf{\Pi}_{w}}=\mathbf{f}_{\mathrm{ref} r}+\left(\frac{d P_{w}}{d t}\right)_{\text {diss }},
$$

where

$$
\overleftrightarrow{\mathbf{\Pi}_{w}}=\int d \mathbf{k} \mathbf{k}\left(\mathbf{V}+\mathbf{v}_{g}\right) N
$$

is the total wave momentum density flux, 


$$
\left(\frac{d P_{w}}{d t}\right)_{\text {diss }}=\int d \mathbf{k} \mathbf{k} C(N)=\int d \mathbf{k} \mathbf{k}\left[C_{w-w}(N)+C_{w-p}(N)\right]
$$

is the total wave momentum dissipated by quasiparticle interactions (N.B. in practice, such momentum dissipation almost always occurs via linear and nonlinear wave-particle momentum transfer), and

$$
\mathbf{f}_{\mathrm{ref} r}=\int d \mathbf{k}\left[-\frac{\partial}{\partial \mathbf{x}}(\omega+\mathbf{k} \cdot \mathbf{V}) N\right]
$$

is the refractive force. As noted in the text for fluctuating strain fields, the wave population density may be further decomposed into a mean and fluctuating piece (i.e., $N=\langle N\rangle$ $+\tilde{N})$, so the mean field refractive force may be written as

$$
\left\langle\mathbf{f}_{\mathrm{ref} r}\right\rangle=-\int d \mathbf{k}\left\{\left(\frac{\partial}{\partial \mathbf{x}}\langle\omega+\mathbf{k} \cdot \mathbf{V}\rangle\right)\langle N\rangle+\overleftrightarrow{\mathbf{D}_{\mathbf{k}}} \cdot \frac{\partial\langle N\rangle}{\partial \mathbf{k}}\right\},
$$

where

$$
\overleftrightarrow{\mathbf{D}_{\mathbf{k}}}=\sum_{\mathbf{q}} \mathbf{q q}\left|(\widetilde{\omega}+\mathbf{k} \cdot \tilde{\mathbf{V}})_{q}\right|^{2} R\left(\Omega-\mathbf{q} \cdot \mathbf{v}_{\mathrm{gr}}\right)
$$

is the $k$-space diffusion tensor for stochastic refraction.

The interpretation of $\left\langle\mathbf{f}_{\mathrm{ref} r}\right\rangle$ as a force density follows from the definition $\partial \mathbf{P}_{w} / \partial t=\left\langle f_{\text {refr }}\right\rangle+\cdots$ and the fact that $d P_{N R} / d t=d P_{W} / d t$. Moreover, $\left\langle f_{\text {ref } r}\right\rangle$ is a local source/sink of momentum, and not a divergence of a flux. The physics of the refractive force is simple-refraction tilts wave packet trajectories, thus changing their momentum. For example, the familiar case of a mean shear flow $\left\langle v_{y}(x)\right\rangle$, the refractive force is just

$$
\left\langle\mathbf{f}_{\text {refr } r}\right\rangle=\left\langle f_{\text {ref }}\right\rangle \hat{\mathbf{x}}=-\frac{\partial v_{y}}{\partial x} \int d \mathbf{k} k_{y}\langle N\rangle .
$$

This corresponds to the familiar cartoon explanation of "zonal flow formation," where "tilting" of wave packets and eddies by coherent shear flows, which diverts $\hat{\mathbf{x}}$-direction momentum into $\hat{\mathbf{y}}$-direction momentum and so applies a force (or stress) on the wave packet, leading to shear flow amplification. Similarly for stochastic (i.e., diffusive) refraction by a spectrum of zonal flows (with $\mathbf{q}=q \hat{\mathbf{x}}$ only),

$$
\left\langle\mathbf{f}_{\text {refr }}\right\rangle=\left\langle f_{\text {refr } r}\right\rangle \hat{\mathbf{x}}=-\int d \mathbf{k} D_{k_{x}, k_{x}} \frac{\partial\langle N\rangle}{\partial k_{x}}
$$

so the refractive force is just the integrated flux in $\mathbf{k}$ produced by random shearing. Here $D_{k_{x}, k_{x}}=\hat{\mathbf{x}} \cdot \overleftrightarrow{\mathbf{D}_{\mathbf{k}}} \cdot \hat{\mathbf{x}}$. Proceeding heuristically, we can immediately note that the power expended by the wave packet against the refractive force is just

$$
P=\mathbf{f} \cdot \mathbf{V} \approx \int d \mathbf{k} v_{\mathrm{gr}} f_{r}=-\int d \mathbf{k} v_{\mathrm{gr}} D_{k_{x}, k_{x}} \frac{\partial\langle N\rangle}{\partial k_{x}} .
$$

This result may be recovered systematically from wavekinetics by taking the energy moment of the wave kinetic equation, $^{72}$ i.e.,

$$
\frac{\partial \varepsilon_{\omega}}{\partial t}=-\int d \mathbf{k} \omega_{\mathbf{k}} \frac{\partial}{\partial \mathbf{k}} \cdot\left[\frac{d \mathbf{k}}{d t} N\right]=\int d \mathbf{k} \mathbf{v}_{g} \cdot \frac{d \mathbf{k}}{d t} N
$$

so for stochastic zonal flow shears

$$
\frac{\partial \varepsilon_{\omega}}{\partial t}=-\int d \mathbf{k} v_{\operatorname{gr}} D_{k_{x}, k_{x}} \frac{\partial\langle N\rangle}{\partial k_{x}}
$$

in agreement with the heuristic argument. Note that here, $N$ is the wave action density. Interestingly, using $N=\Omega$ along with the appropriate corresponding relation $\epsilon_{\omega}=N /(1$ $+k_{\perp}^{2} \rho_{s}^{2}$ ) yields an identical result for $P$, which is the macroscopic observable (i.e., power expended by waves). Since the total energy of waves and zonal flows is conserved up to dissipation, i.e., $d\left(\varepsilon_{\mathrm{Wave}}+\varepsilon_{\mathrm{ZF}}\right) / d t=0,{ }^{43}$ this establishes that the refractive force of random shearing is in fact precisely what does the work to drive zonal flows against collisional dissipation, and that the refractive force may be viewed as the effective body force which drives the zonal flow, thus connecting the refractive force to familiar concepts. Note that a parallel wave group velocity is required to energize a parallel mean flow via the parallel refractive force, just as a radial group velocity is required to energize zonal flows via the radial refractive (i.e., "eddy tilting") force.

Finally, we comment that since the refractive force enters the balance of wave quanta density $N$, wave quanta momentum density $\mathbf{P}_{w}=\mathbf{k} N$, and wave quanta energy density $\varepsilon_{w}=\omega N$ it necessarily must play a role in the dynamics of spreading or propagation of wave intensity $I \sim \int d \mathbf{k} N$, wave momentum density $\mathbf{P}_{w}=\int d \mathbf{k} \mathbf{k} N$, and wave energy density $\varepsilon_{w}=\int d \mathbf{k} \omega N$. It is not clear, however, that the transport of all moments of $N$ will be affected equally. Clearly, the "radiation hydrodynamics" of turbulence spreading and nonlocality phenomena is a promising and interesting topic for future research.

${ }^{1}$ S. D. Scott, P. H. Diamond, R. J. Fonck, R. J. Goldston, R. B. Howell, K. P. Jaehnig, G. Schilling, E. J. Synakowski, M. C. Zarnstorff, C. E. Bush, E. Fredrickson, K. W. Hill, A. C. Janos, D. K. Mansfield, D. K. Owens, H. Park, G. Pautasso, A. T. Ramsey, J. Schivell, G. D. Tait, W. M. Tang, and G. Taylor, Phys. Rev. Lett. 64, 531 (1990).

${ }^{2}$ H. Biglari, P. H. Diamond, and P. W. Terry, Phys. Fluids B 2, 1 (1990).

${ }^{3}$ R. J. Groebner, K. H. Burrell, and R. P. Seraydarian, Phys. Rev. Lett. 64, 3015 (1990).

${ }^{4}$ K. H. Burrell, Phys. Plasmas 4, 1499 (1997).

${ }^{5}$ P. H. Diamond and Y. B. Kim, Phys. Fluids B 3, 1626 (1991).

${ }^{6}$ K. H. Burrell, E. J. Doyle, P. Gohil, R. J. Groebner, J. Kim, R. J. La Haye, L. L. Lao, R. A. Moyer, T. H. Osborne, W. A. Peebles, C. L. Rettig, T. H. Rhodes, and D. M. Thomas, Phys. Plasmas 1, 1536 (1994).

${ }^{7}$ K. Ida, T. Minami, Y. Yoshimura, A. Fujisawa, C. Suzuki, S. Okamura, S. Nishimura, M. Isobe, H. Iguchi, K. Itoh, S. Kado, Y. Liang, I. Nomura, M. Osakabe, C. Takahashi, K. Tanaka, and K. Matsuoka, Phys. Rev. Lett. 86, 3040 (2001).

${ }^{8}$ B. Gonçalves, C. Hidalgo, M. A. Pedrosa, R. O. Orozco, E. Sanchez, and C. Silva, Phys. Rev. Lett. 96, 145001 (2006).

${ }^{9}$ J. E. Rice, M. Greenwald, I. H. Hutchinson, E. S. Marmar, Y. Takase, S. M. Wolfe, and F. Bombarda, Nucl. Fusion 38, 75 (1998).

${ }^{10}$ J. E. Rice, A. Ince-Cushman, J. S. deGrassie, L.-G. Eriksson, Y. Sakamoto, A. Scarabosio, A. Bortolon, K. H. Burrell, C. Fenzi-Bonizec, M. J. Greenwald, R. J. Groebner, G. T. Hoang, Y. Koide, E. S. Marmar, A. Pochelon, and Y. Podpaly, 21th IAEA Fusion Energy Conference, Chengdu, China (International Atomic Energy Agency, Vienna, 2006), pp. EX/p3-12.

${ }^{11}$ J. S. deGrassie, J. E. Rice, K. H. Burrell, R. J. Groebner, and W. M. Solomon, Phys. Plasmas 14, 056115 (2007).

${ }^{12}$ M. Yoshida, Y. Koide, H. Takenaga, H. Urano, N. Oyama, K. Kamiya, Y. 
Sakamoto, and Y. Kamada, Plasma Phys. Controlled Fusion 48, 1673 (2006).

${ }^{13}$ W. M. Solomon, K. H. Burrell, J. S. deGrassie, R. Budny, R. J. Groebner, J. E. Kinsey, G. J. Kramer, M. A. Makowski, D. Mikkelsen, R. Nazikian, C. C. Petty, P. A. Politzer, S. D. Scott, M. A. Van Zeeland, and M. C. Zarnstorff, "Momentum confinement at low torque," Plasma Phys. Controlled Fusion (in press).

${ }^{14}$ J. E. Rice, E. S. Marmar, P. T. Bonoli, R. S. Granetz, M. J. Greenwald, A. E. Hubbard, J. W. Hughes, I. H. Hutchinson, J. H. Irby, B. Labombard, W. D. Lee, Y. Lin, D. Mossessian, J. A. Snipes, S. M. Wolfe, and S. J. Wukitch, Fusion Sci. Technol. 51, 288 (2007).

${ }^{15}$ B. LaBombard, J. E. Rice, A. E. Hubbard, J. W. Hughes, M. Greenwald, J. Irby, Y. Lin, B. Lipschultz, E. S. Marmar, C. S. Pitcher, N. Smick, S. M. Wolfe, and S. J. Wukitch, Nucl. Fusion 44, 1047 (2004).

${ }^{16}$ A. Bortolon, B. P. Duval, A. Pochelon, and A. Scarabosio, Phys. Rev. Lett. 97, 235003 (2006)

${ }^{17}$ M. Yoshida, Y. Koide, H. Takenaga, H. Urago, N. Oyama, K. Kamiya, Y. Sakamoto, Y. Kamada, and the JT-60 Team, in Proceedings of the 21th IAEA Fusion Energy Conference, Chengdu, China (International Atomic Energy Agency, Vienna, 2006), pp. IAEA/EX-P3-22.

${ }^{18}$ P. Monier-Garbet, P. Andrew, P. Belo, G. Bonheure, Y. Corre, K. Crombe, P. Dumortier, T. Eich, R. Felton, J. Harling, J. Hogan, A. Huber, S. Jaclunich, E. Joffrin, H. R. Koslowski, A. Kreter, G. Maddison, G. F. Matthews, A. Messiaen, M. F. Nave, J. Ongena, V. Parail, M. E. Puiatti, J. Rapp, R. Sartori, J. Stober, M. Z. Tokar, B. Unterberg, M. Valisa, I. Voitsekhovitch, and M. von Hellerman, Phys. Rev. Lett. 95, 155003 (2005).

${ }^{19}$ S. I. Itoh, Phys. Fluids B 4, 796 (1992).

${ }^{20}$ N. Mattor and P. H. Diamond, Phys. Fluids 31, 1180 (1988).

${ }^{21}$ K. C. Shaing, Phys. Plasmas 8, 193 (2001).

${ }^{22}$ A. G. Peeters, C. Angioni, and D. Strintzi, Phys. Rev. Lett. 98, 265003 (2007).

${ }^{23}$ T. S. Hahm, P. H. Diamond, O. D. Gurcan, and G. Rewoldt, Phys. Plasmas 14, 072302 (2007)

${ }^{24}$ Ö. D. Gürcan, P. H. Diamond, and T. S. Hahm, "Turbulent equipartition and homogenization of angular momentum," Phys. Rev. Lett. (submitted).

${ }^{25}$ Ö. D. Gürcan, P. H. Diamond, T. S. Hahm, and R. Singh, Phys. Plasmas 14, 042306 (2007).

${ }^{26}$ Y. B. Kim, P. H. Diamond, H. Biglari, and P. W. Terry, Phys. Fluids B 2, 2143 (1990)

${ }^{27}$ R. R. Dominguez and G. M. Staebler, Phys. Fluids B 5, 3876 (1993).

${ }^{28}$ P. H. Diamond, V. B. Lebedev, Y. M. Liang, A. V. Gruzinov, I. Gruzinova, and M. Medvedev, in Proceedings of the 15th IAEA Fusion Energy Conference, Seville, Spain (International Atomic Energy Agency, Vienna, 1994), pp. IAEA-CN-60/D-2-II-6.

${ }^{29}$ X. Garbet, Y. Sarazin, P. Ghendrih, S. Benkadda, P. Beyer, C. Figarella, and I. Voitsekhovitch, Phys. Plasmas 9, 3893 (2002).

${ }^{30}$ J. R. Myra, J. Boedo, B. Coppi, D. A. D’Ippolito, S. I. Krasheninnikov, B. P. LeBlanc, M. Lontano, R. Maqueda, D. A. Russell, D. P. Stotler, M. C. Varishetti, S. J. Zweben, and the NSTX Team, in Proceedings of the 21th IAEA Fusion Energy Conference, Chengdu, China (International Atomic Energy Agency, Vienna, 2006), pp. IAEA/TH-P6-21.

${ }^{31}$ L. Chen, J. Geophys. Res. 104, 2421, DOI: 10.1029/1998JA900051 (1999).

${ }^{32}$ R. Z. Sagdeev and A. A. Galeev, Nonlinear Plasma Theory (Benjamin, New York, 1969).

${ }^{33}$ D. Mihalas and B. Weibel-Mihalas, Foundations of Radiation Hydrodynamics (Oxford University Press, Oxford, 1984).

${ }^{34}$ X. Garbet, L. Laurent, A. Samain, and J. Chinardet, Nucl. Fusion 34, 963 (1994).
${ }^{35}$ Z. Lin and T. S. Hahm, Phys. Plasmas 11, 1099 (2004).

${ }^{36}$ T. S. Hahm, P. H. Diamond, Z. Lin, K. Itoh, and S. I. Itoh, Plasma Phys. Controlled Fusion 46, A323 (2004).

${ }^{37}$ Ö. D. Gürcan, P. H. Diamond, and T. S. Hahm, Phys. Rev. Lett. 97, 024502 (2006).

${ }^{38}$ M. N. Rosenbluth and C. S. Liu, Phys. Fluids 19, 815 (1976).

${ }^{39}$ P. H. Diamond and T. S. Hahm, Phys. Plasmas 2, 3640 (1995).

${ }^{40}$ B. A. Carreras, D. Newman, V. E. Lynch, and P. H. Diamond, Phys. Plasmas 3, 2903 (1996).

${ }^{41}$ P. H. Diamond and Y.-B. Kim, Phys. Fluids B 3, 1626 (1991).

${ }^{42}$ H. L. Berk and K. Molvig, Phys. Fluids 26, 1385 (1983).

${ }^{43}$ P. H. Diamond, S. I. Itoh, K. Itoh, and T. S. Hahm, Plasma Phys. Controlled Fusion 47, R35 (2005).

${ }^{44}$ P. H. Diamond, M. N. Rosenbluth, F. L. Hinton, M. Malkov, J. Fleischer, and A. Smolyakov, in Proceedings of the 17th IAEA Fusion Energy Conference, Yokohama, Japan (International Atomic Energy Agency, Vienna, 1998), pp. IAEA-CN-69/TH3/1.

${ }^{45}$ F. P. Bretherton, J. Fluid Mech. 36, 785 (1969).

${ }^{46}$ S. V. Nazarenko, N. J. Zabusky, and T. Scheidegger, Phys. Fluids 7, 2407 (1995).

${ }^{47}$ O. Buhler and M. E. McIntyre, J. Fluid Mech. 492, 207 (2003).

${ }^{48}$ G. B. Whitham, Linear and Nonlinear Waves (Wiley-Interscience, New York, 1974).

${ }^{49}$ A. M. Balk, S. V. Nazarenko, and V. E. Zakharov, Phys. Lett. A 146, 217 (1990).

${ }^{50}$ A. I. Smolyakov and P. H. Diamond, Phys. Plasmas 6, 4410 (1999).

${ }^{51}$ B. Dubrulle and S. Nazarenko, Physica D 110, 123 (1997).

${ }^{52}$ J. R. Pierce, Travelling Wave Tubes (Van Nostrand, New York, 1950).

${ }^{53}$ C. H. McComas and F. P. Bretherton, J. Geophys. Res. 82, 1367 (1977).

${ }^{54}$ V. E. Zakharov, V. S. Lvov, and G. Falkovich, Kolmogorov Spectra of Turbulence I: Wave Turbulence (Springer-Verlag, Berlin, 1992).

${ }^{55}$ W. M. Manheimer and T. H. Dupree, Phys. Fluids 11, 2709 (1968).

${ }^{56}$ E. M. Lifshitz and L. P. Pitaevskii, Physical Kinetics (ButterworthHeinemann, London, 1981).

${ }^{57}$ C. P. Ritz, H. Lin, T. L. Rhodes, and A. J. Wootton, Phys. Rev. Lett. 65, 2543 (1990).

${ }^{58}$ B. B. Kadomtsev, Plasma Phys. Controlled Fusion 34, 1931 (1992).

${ }^{59}$ E. A. Spiegel, Astrophys. J. 138, 216 (1963).

${ }^{60}$ M. S. Longuet-Higgins and R. W. Stewart, Deep-Sea Res. 11, 529 (1964).

${ }^{61}$ N. Mattor and P. H. Diamond, Phys. Rev. Lett. 72, 486 (1994).

${ }^{62}$ J. Q. Dong, W. H. R. D. Bengtson, and G. X. Li, Phys. Plasmas 1, 3250 (1994).

${ }^{63}$ B. Coppi, Nucl. Fusion 42, 1 (2002).

${ }^{64} \mathrm{~J}$. E. Rice (private communication, 2006).

${ }^{65}$ J. D. Murray, Mathematical Biology, 2nd ed. (Springer-Verlag, Berlin, 1993).

${ }^{66}$ L. D. Landau, E. M. Lifshitz, and L. P. Pitaevskii, Electrodynamics of Continuous Media (Pergamon, Oxford, 1981).

${ }^{67}$ C. E. Leith, Phys. Fluids 11, 671 (1968).

${ }^{68}$ C. Connaughton and S. Nazarenko, Phys. Rev. Lett. 92, 044501 (2004).

${ }^{69}$ X. Garbet, Y. Sarazin, F. Imbeaux, P. Ghendrih, C. Bourdelle, O. D. Gurcan, and P. H. Diamond, Phys. Plasmas 14, 122305 (2007).

${ }^{70}$ V. Naulin, A. H. Nielsen, and J. J. Rasmussen, Phys. Plasmas 12, 122306 (2005).

${ }^{71}$ J. Krommes, Phys. Fluids B 5, 1066 (1993).

${ }^{72}$ P. H. Diamond, M. N. Rosenbluth, E. Sanchez, C. Hidalgo, B. van Milligen, T. Estrada, B. Branas, M. Hirsch, H. J. Hartfuss, and B. A. Carreras, Phys. Rev. Lett. 84, 4842 (2000). 